\title{
HARMONIC SPHERES IN OUTER SYMMETRIC SPACES, THEIR CANONICAL ELEMENTS AND WEIERSTRASS-TYPE REPRESENTATIONS
}

\author{
N. CORREIA AND R. PACHECO
}

\begin{abstract}
Making use of Murakami's classification of outer involutions in a Lie algebra and following the Morse-theoretic approach to harmonic two-spheres in Lie groups introduced by Burstall and Guest, we obtain a new classification of harmonic two-spheres in outer symmetric spaces and a Weierstrass-type representation for such maps. Several examples of harmonic maps into classical outer symmetric spaces are given in terms of meromorphic functions on $S^{2}$.
\end{abstract}

\section{INTRODUCTION}

The harmonicity of maps $\varphi$ from a Riemann surface $M$ into a compact Lie group $G$ with identity $e$ amounts to the flatness of one-parameter families of connections. This establishes a correspondence between such maps and certain holomorphic maps $\Phi$ into the based loop group $\Omega G$, the extended solutions [17. Evaluating an extended solution $\Phi$ at $\lambda=-1$ we obtain a harmonic map $\varphi$ into the Lie group. If an extended solution takes values in the group of algebraic loops $\Omega_{\mathrm{alg}} G$, the corresponding harmonic map is said to have finite uniton number. It is well known that all harmonic maps from the two-sphere into a compact Lie group have finite uniton number [17.

Burstall and Guest [1] have used a method suggested by Morse theory in order to describe harmonic maps with finite uniton number from $M$ into a compact Lie group $G$ with trivial centre. One of the main ingredients in that paper is the Bruhat decomposition of the group of algebraic loops $\Omega_{\mathrm{alg}} G$. Each piece $U_{\xi}$ of the Bruhat decomposition corresponds to an element $\xi$ in the integer lattice $\Im(G)=(2 \pi)^{-1} \exp ^{-1}(e) \cap \mathfrak{t}$ and can be described as the unstable manifold of the energy flow on the Kähler manifold $\Omega_{\text {alg }} G$. Each extended solution $\Phi: M \rightarrow \Omega_{\text {alg }} G$ takes values, off some discrete subset $D$ of $M$, in one of these unstable manifolds $U_{\xi}$ and can be deformed, under the gradient flow of the energy, to an extended solution with values in some conjugacy class of a Lie group homomorphism $\gamma_{\xi}: S^{1} \rightarrow G$. A normalization procedure allows us to choose $\xi$ among the canonical elements of $\mathfrak{I}(G)$; there are precisely $2^{n}$ canonical elements, where $n=\operatorname{rank}(G)$, and consequently $2^{n}$ classes of harmonic maps. Burstall and Guest [1] introduced also a Weierstrass-type representation for such harmonic maps in terms of meromorphic functions on $M$. It is possible to define a similar notion of canonical element for compact Lie groups $G$ with non-trivial centre [5, 6. In the present paper, we will not assume any restriction on the centre of $G$.

Given an involution $\sigma$ of $G$, the compact symmetric $G$-space $N=G / G^{\sigma}$, where $G^{\sigma}$ is the subgroup of $G$ fixed by $\sigma$, can be embedded totally geodesically in $G$ via the corresponding Cartan embedding $\iota_{\sigma}$. Hence harmonic maps into compact symmetric spaces can be interpreted as special harmonic maps into Lie groups. For inner involutions $\sigma=\operatorname{Ad}\left(s_{0}\right)$, where $s_{0} \in G$ is the geodesic reflection at some base point $x_{0} \in N$, the composition of the Cartan embedding with left multiplication by $s_{0}$ gives a totally geodesic embedding of $G / G^{\sigma}$ in $G$ as a connected component of $\sqrt{e}$. Reciprocally, any connected component of $\sqrt{e}$ is a compact inner symmetric $G$-space. As shown by Burstall and Guest [1, any harmonic map into a connected component of $\sqrt{e}$ admits an extended solution $\Phi$ which is invariant under the involution $I(\Phi)(\lambda)=\Phi(-\lambda) \Phi(-1)^{-1}$. Off a discrete set, $\Phi$ takes values in some unstable manifold $U_{\xi}$ and can be deformed, under the gradient flow of the energy, to an extended solution with values in some conjugacy class of a Lie group homomorphism $\gamma_{\xi}: S^{1} \rightarrow G^{\sigma}$. An appropriate normalization procedure which preserves both $I$-invariance and the underlying 
N. CORREIA AND R. PACHECO

connected component of $\sqrt{e}$ allows us to choose $\xi$ among the canonical elements of $\mathfrak{I}\left(G^{\sigma}\right)$. As a matter of fact, since $\sigma$ is inner, $\operatorname{rank}(G)=\operatorname{rank}\left(G^{\sigma}\right)$ and we have $\mathfrak{I}(G)=\mathfrak{I}\left(G^{\sigma}\right)$, that is the canonical elements of $\mathfrak{I}(G)$ coincide with the canonical elements of $\mathfrak{I}\left(G^{\sigma}\right)$. Consequently, if $G$ has trivial center, we have $2^{n}$ classes of harmonic maps with finite uniton number into all inner symmetric $G$-spaces.

The theory of Burstall and Guest [1] on harmonic two-spheres in compact inner symmetric $G$-spaces was extended by Eschenburg, Mare and Quast [8] to outer symmetric spaces as follows: each harmonic map from a two-sphere into an outer symmetric space $G / G^{\sigma}$, with outer involution $\sigma$, corresponds to an extended solution $\Phi$ which is invariant under a certain involution $T_{\sigma}$ induced by $\sigma$ on $\Omega G$ (see also [11); $\Phi$ takes values in some unstable manifold $U_{\xi}$, off some discrete set; under the gradient flow of the energy any such invariant extended solution is deformed to an extended solution with values in some conjugacy class of a Lie group homomorphism $\gamma_{\xi}: S^{1} \rightarrow G^{\sigma}$; applying the normalization procedure of extended solutions introduced by Burstall and Guest for Lie groups, $\xi$ can be chosen among the canonical elements of $\mathfrak{I}\left(G^{\sigma}\right) \subsetneq \Im(G)$; if $G$ has trivial centre, there are precisely $2^{k}$ canonical homorphisms, where $k=\operatorname{rank}\left(G^{\sigma}\right)<\operatorname{rank}(G)$; hence there are at most $2^{k}$ classes of harmonic two-spheres in $G / G^{\sigma}$ if $G$ has trivial centre.

In the present paper, we will establish a more accurate classification of harmonic maps from a two-sphere into compact outer symmetric spaces. This classification takes into consideration the following crucial facts concerning extended solutions associated to harmonic maps into outer symmetric spaces: although any harmonic map from a two-sphere into an outer symmetric space $G / G^{\sigma}$ admits a $T_{\sigma}$-invariant extended solution, not all $T_{\sigma}$-invariant extended solutions correspond to harmonic maps into $G / G^{\sigma}$; on the other hand, the Burstall and Guest's normalization procedure does not necessarily preserve $T_{\sigma}$-invariance.

Our strategy is the following. The existence of outer involutions of a simple Lie algebra $\mathfrak{g}$ depends on the existence of non-trivial involutions of the Dynkin diagram of $\mathfrak{g}^{\mathbb{C}}[2,8,12$, 14. More precisely, if $\varrho$ is a nontrivial involution of the Dynkin diagram of $\mathfrak{g}^{\mathbb{C}}$, then it induces an outer involution $\sigma_{\varrho}$ of $\mathfrak{g}^{\mathbb{C}}$, which we call the fundamental outer involution, and, as shown by Murakami [14, all the other outer involutions are, up to conjugation, of the form $\sigma_{\varrho, i}:=\operatorname{Ad} \exp \pi \zeta_{i} \circ \sigma_{\varrho}$ where each $\zeta_{i}$ is a certain element in the integer lattice $\mathfrak{I}\left(G^{\sigma_{\varrho}}\right)$. Each connected component of $P^{\sigma_{\varrho}}=\left\{g \in G \mid \sigma_{\varrho}(g)=g^{-1}\right\}$ is a compact outer symmetric $G$-space associated to some involution $\sigma_{\varrho}$ or $\sigma_{\varrho, i}$; reciprocally, any outer symmetric space $G / G^{\sigma}$, with $\sigma$ equal to $\sigma_{\varrho}$ or $\sigma_{\varrho, i}$, can be totally geodesically embedded in the Lie group $G$ as a connected component of $P^{\sigma_{e}}$ (see Proposition [6). As shown in Section 4.2, any harmonic map $\varphi$ into a connected component $N$ of $P^{\sigma_{Q}}$ admits a $T_{\sigma_{e}}$-invariant extended solution $\Phi$; off a discrete set, $\Phi$ takes values in some unstable manifold $U_{\xi}$. In Section 4.2 .2 we introduce an appropriate normalization procedure in order to obtain from $\Phi$ a normalized extended solution $\tilde{\Phi}$ with values in some unstable manifold $U_{\zeta}$ such that: $\zeta$ is a canonical element of $\Im\left(G^{\sigma_{\varrho}}\right)$; $\tilde{\Phi}$ is $T_{\tau}$-invariant, where $\tau$ is the outer involution given by $\tau=\operatorname{Ad} \exp \pi(\xi-\zeta) \circ \sigma_{\varrho} ; \tilde{\Phi}(-1)$ takes values in some connected component of $P^{\sigma_{e}}$ which is an isometric copy of $N$ completely determined by $\zeta$ and $\tau$; moreover, $\tilde{\Phi}(-1)$ coincides with $\varphi$ up to isometry. Hence, we obtain a classification of harmonic maps of finite uniton number from $M$ into outer symmetric $G$-spaces in terms of the pairs $(\zeta, \tau)$.

Dorfmeister, Pedit and $\mathrm{Wu}$ [7] have introduced a general scheme for constructing harmonic maps from a Riemann surface into a compact symmetric space from holomorphic data, in which the harmonic map equation reduces to a linear ODE similar to the classical Weierstrass representation of minimal surfaces. Burstal and Guest 1 made this scheme more explicit for the case $M=S^{2}$ by establishing a "Weierstrass formula" for harmonic maps with finite uniton number into Lie groups and their inner symmetric spaces. In Theorem 17 we establish a version of this formula to outer symmetric spaces, which allows us to describe the corresponding $T_{\sigma}$-invariant extended solutions in terms of meromorphic functions on $M$. For normalized extended solutions and "low uniton number", such descriptions are easier to obtain. In Section 5 we give several explicit examples of harmonic maps from the two-sphere into classical outer symmetric spaces: Theorem 20 interprets old results by Calabi [3] and Eells and Wood [9] concerning harmonic spheres in real projective spaces $\mathbb{R} P^{2 n-1}$ in view of our classification; harmonic two-spheres into the real Grassmannian $G_{3}\left(\mathbb{R}^{6}\right)$ are studied in detail; we show that all harmonic two spheres into the Wu manifold $S U(3) / S O(3)$ can be obtained explicitly by choosing two 
meromorphic functions on $S^{2}$ and then performing a finite number of algebraic operations, in agreement with the explicit constructions established by H. Ma in [13.

\section{Groups of Algebraic LOOPS}

For completeness, in this section we recall some fundamental facts concerning the structure of the group of algebraic loops in a compact Lie group. Further details can be found in [1, 4, 15].

Let $G$ be a compact matrix semisimple Lie group with Lie algebra $\mathfrak{g}$ and identity $e$. Denote the free and based loop groups of $G$ by $\Lambda G$ and $\Omega G$, respectively, whereas $\Lambda_{+} G^{\mathbb{C}}$ stands for the subgroup of $\Lambda G^{\mathbb{C}}$ consisting of loops $\gamma: S^{1} \rightarrow G^{\mathbb{C}}$ which extend holomorphically to the unitary disc $|\lambda|<1$.

Fix a maximal torus $T$ of $G$ with Lie algebra $\mathfrak{t} \subset \mathfrak{g}$. Let $\Delta \subset$ it* $^{*}$ be the corresponding set of roots, where $\mathrm{i}:=\sqrt{-1}$, and, for each $\alpha \in \Delta$, denote by $\mathfrak{g}_{\alpha}$ the corresponding root space. Choose a fundamental Weyl chamber $\mathcal{W}$ in $\mathfrak{t}$, which corresponds to fix a positive root system $\Delta^{+}$. The intersection $\mathfrak{I}^{\prime}(G):=\mathfrak{I}(G) \cap \mathcal{W}$ parameterizes the conjugacy classes of homomorphisms $S^{1} \rightarrow G$. More precisely, $\operatorname{Hom}\left(S^{1}, G\right)$ is the disjoint union of $\Omega_{\xi}(G)$, with $\xi \in \mathfrak{I}^{\prime}(G)$, where $\Omega_{\xi}(G)$ is the conjugacy class of homomorphisms which contains $\gamma_{\xi}(\lambda)=\exp (-\mathrm{i} \ln (\lambda) \xi)$.

The Bruhat decomposition states that the subgroup of algebraic based loops $\Omega_{\text {alg }} G$ is the disjoint union of the orbits $\Lambda_{\text {alg }}^{+} G^{\mathbb{C}} \cdot \gamma_{\xi}$, with $\xi \in \mathfrak{I}^{\prime}(G)$, where $\cdot$ denotes the dressing action of $\Lambda_{+} G^{\mathbb{C}}$ on $\Omega G$ induced by the Iwasawa decomposition $\Lambda G^{\mathbb{C}} \cong \Omega G \times \Lambda_{+} G^{\mathbb{C}}$. According to the Morse theoretic interpretation [1, 15] of the Bruhat decomposition, for each $\xi \in \mathfrak{I}^{\prime}(G), U_{\xi}(G):=\Lambda_{\text {alg }}^{+} G^{\mathbb{C}} \cdot \gamma_{\xi}$ is the unstable manifold of $\Omega_{\xi}(G)$ under the flow induced by the energy gradient vector field $-\nabla E$ : each $\gamma \in U_{\xi}(G)$ flows to some homomorphism $u_{\xi}(\gamma)$ in $\Omega_{\xi}(G)$.

In 11, the authors proved that, for each $\xi \in \mathfrak{I}^{\prime}(G)$, the critical manifold $\Omega_{\xi}(G)$ is a complex homogeneous space of $G^{\mathbb{C}}$ and the unstable manifold $U_{\xi}(G)$ is a complex homogeneous space of the group $\Lambda_{\text {alg }}^{+} G^{\mathbb{C}}$. Moreover, $U_{\xi}(G)$ carries a structure of holomorphic vector bundle over $\Omega_{\xi}(G)$ and the bundle map $u_{\xi}: U_{\xi}(G) \rightarrow \Omega_{\xi}(G)$ is precisely the natural projection given by $[\gamma] \mapsto[\gamma(0)]$.

Define a partial order $\preceq$ over $\mathfrak{I}^{\prime}(G)$ as follows: $\xi \preceq \xi^{\prime}$ if $\mathfrak{p}_{i}^{\xi} \subset \mathfrak{p}_{i}^{\xi^{\prime}}$ for all $i \geq 0$, where $\mathfrak{p}_{i}^{\xi}=\sum_{j \leq i} \mathfrak{g}_{j}^{\xi}$ and $\mathfrak{g}_{j}^{\xi}$ is the $j$ i-eigenspace of ad $\xi$. As shown in [4, one can define a $\Lambda_{\text {alg }}^{+} G^{\mathbb{C}}$-invariant fibre bundle morphism $\mathcal{U}_{\xi, \xi^{\prime}}: U_{\xi}(G) \rightarrow U_{\xi^{\prime}}(G)$ by

$$
\mathcal{U}_{\xi, \xi^{\prime}}\left(\Psi \cdot \gamma_{\xi}\right)=\Psi \cdot \gamma_{\xi^{\prime}}, \quad \Psi \in \Lambda_{\text {alg }}^{+} G^{\mathbb{C}}
$$

whenever $\xi \preceq \xi^{\prime}$. Since the holomorphic structures on $U_{\xi}(G)$ and $U_{\xi^{\prime}}(G)$ are induced by the holomorphic structure on $\Lambda_{\text {alg }}^{+} G^{\mathbb{C}}$, the fibre-bundle morphism $\mathcal{U}_{\xi, \xi^{\prime}}$ is holomorphic.

\section{HARMONIC SPHERES IN LIE GROUPS}

Harmonic maps from the two-sphere $S^{2}$ into a compact matrix Lie group $G$ can be classified in terms of certain pieces of the Bruhat decomposition of $\Omega_{\mathrm{alg}} G$. Next we recall briefly this theory from [1, 4, 5, 6,

3.1. Extended Solutions. Let $M$ be a simply-connected Riemann surface, $\varphi: M \rightarrow G$ be a smooth map and $\rho: G \rightarrow \operatorname{End}(V)$ a finite representation of $G$. Equip $G$ with a bi-invariant metric. If $\varphi$ is an harmonic map of finite uniton number, it admits an extended solution $\Phi: M \rightarrow \Omega G$ with $\Phi(M) \subseteq \Omega_{\text {alg }} G$ and $\varphi=\Phi_{-1}$. In this case, we can write $\rho \circ \Phi=\sum_{i=r}^{s} \zeta_{i} \lambda^{i}$ for some $r \leq s \in \mathbb{Z}$. The number $s-r$ is called the uniton number of $\Phi$ with respect to $\rho$, and the minimal value of $s-r$ (with respect to all extended solutions associated to $\varphi$ ) is called the uniton number of $\varphi$ with respect to $\rho$ and it is denoted by $r_{\rho}(\varphi)$. As explained in [6], this definition of uniton number of an extended solution with respect to the adjoint representation is twice that of Burstall and Guest [1. K. Uhlenbeck [17] proved that all harmonic maps from the two-sphere have finite uniton number. For simplicity of exposition, henceforth we will take $M=S^{2}$. However, all our results still hold for harmonic maps of finite uniton number from an arbitrary Riemann surface.

Theorem 1. 1] Let $\Phi: S^{2} \rightarrow \Omega_{\mathrm{alg}} G$ be an extended solution. Then there exists some $\xi \in \mathfrak{I}^{\prime}(G)$, and some discrete subset $D$ of $S^{2}$, such that $\Phi\left(S^{2} \backslash D\right) \subseteq U_{\xi}(G)$. 
Given a smooth map $\Phi: S^{2} \backslash D \rightarrow U_{\xi}(G)$, consider $\Psi: S^{2} \backslash D \rightarrow \Lambda_{\text {alg }}^{+} G^{\mathbb{C}}$ such that $\Phi=\Psi \cdot \gamma_{\xi}$, that is $\Psi \gamma_{\xi}=\Phi b$ for some $b: S^{2} \backslash D \rightarrow \Lambda_{\text {alg }}^{+} G^{\mathbb{C}}$. Write $\Psi^{-1} \Psi_{z}=\sum_{i \geq 0} X_{i}^{\prime} \lambda^{i}$, and $\Psi^{-1} \Psi_{\bar{z}}=\sum_{i \geq 0} X_{i}^{\prime \prime} \lambda^{i}$. Proposition 4.4 in [1] establishes that $\Phi$ is an extended solution if, and only if,

$$
\operatorname{Im} X_{i}^{\prime} \subset \mathfrak{p}_{i+1}^{\xi}, \quad \operatorname{Im} X_{i}^{\prime \prime} \subset \mathfrak{p}_{i}^{\xi},
$$

where $\mathfrak{p}_{i}^{\xi}=\bigoplus_{j \leq i} \mathfrak{g}_{j}^{\xi}$ and $\mathfrak{g}_{j}^{\xi}$ is the $j$ i-eigenspace of ad $\xi$. The derivative of the harmonic map $\varphi=\Phi_{-1}$ is given by the following formula.

Lemma 2. 4] Let $\Phi=\Psi \cdot \gamma_{\xi}: S^{2} \rightarrow \Omega_{\mathrm{alg}} G$ be an extended solution and $\varphi=\Phi_{-1}: S^{2} \rightarrow G$ the corresponding harmonic map. Then

$$
\varphi^{-1} \varphi_{z}=-2 \sum_{i \geq 0} b(0) X_{i}^{i+1} b(0)^{-1}
$$

where $X_{i}^{\prime i+1}$ is the component of $X_{i}^{\prime}$ over $\mathfrak{g}_{i+1}^{\xi}$, with respect to the decomposition $\mathfrak{g}^{\mathbb{C}}=\bigoplus \mathfrak{g}_{j}^{\xi}$.

Both the fiber bundle morphism $\mathcal{U}_{\xi, \xi^{\prime}}: U_{\xi}(G) \rightarrow U_{\xi^{\prime}}(G)$ and the bundle map $u_{\xi}: U_{\xi}(G) \rightarrow \Omega_{\xi}(G)$ preserve harmonicity.

Proposition 3. 1, 4] Let $\Phi: S^{2} \backslash D \rightarrow U_{\xi}(G)$ be an extended solution. Then

a) $u_{\xi} \circ \Phi: S^{2} \backslash D \rightarrow \Omega_{\xi}(G)$ is an extended solution, with $\xi \in \mathfrak{I}(G)$;

b) for each $\xi^{\prime} \in \mathfrak{I}^{\prime}(G)$ such that $\xi \preceq \xi^{\prime}, \mathcal{U}_{\xi, \xi^{\prime}}(\Phi)=\mathcal{U}_{\xi, \xi^{\prime}} \circ \Phi: S^{2} \backslash D \rightarrow U_{\xi^{\prime}}(G)$ is an extended solution.

3.2. Weierstrass representation. Taking a larger discrete subset if necessary, one obtains a more explicit description for harmonic maps of finite uniton number and their extended solutions as follows.

Proposition 4. [1] Let $\Phi: S^{2} \rightarrow \Omega_{\mathrm{alg}} G$ be an extended solution. There exists a discrete set $D^{\prime} \supseteq D$ of $S^{2}$ such that $\left.\Phi\right|_{S^{2} \backslash D^{\prime}}=\exp C \cdot \gamma_{\xi}$ for some holomorphic vector-valued function $C: S^{2} \backslash D^{\prime} \rightarrow \mathfrak{u}_{\xi}^{0}$, where $\mathfrak{u}_{\xi}^{0}$ is the finite dimensional nilpotent subalgebra of $\Lambda_{\text {alg }}^{+} \mathfrak{g}^{\mathbb{C}}$ defined by

$$
\mathfrak{u}_{\xi}^{0}=\bigoplus_{0 \leq i<r(\xi)} \lambda^{i}\left(\mathfrak{p}_{i}^{\xi}\right)^{\perp}, \quad\left(\mathfrak{p}_{i}^{\xi}\right)^{\perp}=\bigoplus_{i<j \leq r(\xi)} \mathfrak{g}_{j}^{\xi},
$$

with $r(\xi)=\max \left\{j \mid \mathfrak{g}_{j}^{\xi} \neq 0\right\}$. Moreover, $C$ can be extended meromorphically to $S^{2}$.

Conversely, taking account (1) and the well-known formula for the derivative of the exponential map, we see that if $C: S^{2} \rightarrow \mathfrak{u}_{\xi}^{0}$ is meromorphic then $\Phi=\exp C \cdot \gamma_{\xi}$ is an extended solution if and only if in the expression

$$
(\exp C)^{-1}(\exp C)_{z}=C_{z}-\frac{1}{2 !}(\operatorname{ad} C) C_{z}+\ldots+(-1)^{r(\xi)-1} \frac{1}{r(\xi) !}(\operatorname{ad} C)^{r(\xi)-1} C_{z},
$$

the coefficient $\lambda^{i}$ have zero component in each $\mathfrak{g}_{i+2}^{\xi}, \ldots, \mathfrak{g}_{r(\xi)}^{\xi}$.

3.3. $S^{1}$-invariant extended solutions. Extended solutions with values in some $\Omega_{\xi}(G)$, off a discrete subset, are said to be $S^{1}$-invariant. If we take a unitary representation $\rho: G \rightarrow U(n)$ for some $n$, then for any such extended solution $\Phi$ we have $\rho \circ \Phi_{\lambda}=\sum_{i=r}^{s} \lambda^{i} \pi_{W_{i}}$, where, for each $i, \pi_{W_{i}}$ is the orthogonal projection onto a complex vector subbundle $W_{i}$ of $\underline{\mathbb{C}}^{n}:=M \times \mathbb{C}^{n}$ and $\underline{\mathbb{C}}^{n}=\bigoplus_{i=r}^{s} W_{i}$ is an orthogonal direct sum decomposition. Set $A_{i}=\bigoplus_{j \leq i} W_{j}$ so that

$$
\{0\} \subset A_{r} \subset \ldots \subset A_{i-1} \subset A_{i} \subset A_{i+1} \subset \ldots \subset A_{s}=\underline{\mathbb{C}}^{n} .
$$

The harmonicity condition amounts to the following conditions on this flag: for each $i, A_{i}$ is a holomorphic subbundle of $\underline{\mathbb{C}}^{n}$; the flag is superhorizontal, in the sense that, for each $i$, we have $\partial A_{i} \subseteq A_{i+1}$, that is, given any section $s$ of $A_{i}$ then $\frac{\partial s}{\partial z}$ is a section of $A_{i+1}$ for any local complex coordinate $z$ of $S^{2}$. 
3.4. Normalization of harmonic maps. Let $\Delta_{0}:=\left\{\alpha_{1}, \ldots, \alpha_{r}\right\} \subset \Delta^{+}$be the basis of positive simple roots, with dual basis $\left\{H_{1}, \ldots, H_{r}\right\} \subset \mathfrak{t}$, that is $\alpha_{i}\left(H_{j}\right)=\mathrm{i} \delta_{i j}$, where $r=\operatorname{rank}(\mathfrak{g})$. Given $\xi=\sum n_{i} H_{i}$ and $\xi^{\prime}=\sum n_{i}^{\prime} H_{i}$ in $\mathfrak{I}^{\prime}(G)$, we have $n_{i}, n_{i}^{\prime} \geq 0$ and observe that $\xi \preceq \xi^{\prime}$ if and only if $n_{i}^{\prime} \leq n_{i}$ for all $i$. For each $I \subseteq\{1, \ldots, r\}$, define the cone $\mathfrak{C}_{I}=\left\{\sum_{i=1}^{r} n_{i} H_{i} \mid n_{i} \geq 0, n_{j}=0\right.$ iff $\left.j \notin I\right\}$.

Definition 1. 6] Let $\xi \in \mathfrak{I}^{\prime}(G) \cap \mathfrak{C}_{I}$. We say that $\xi$ is a $I$-canonical element of $G$ with respect to $\mathcal{W}$ if it is a maximal element of $\left(\mathfrak{I}^{\prime}(G) \cap \mathfrak{C}_{I}, \preceq\right)$, that is: if $\xi \preceq \xi^{\prime}$ and $\xi^{\prime} \in \mathfrak{I}^{\prime}(G) \cap \mathfrak{C}_{I}$ then $\xi=\xi^{\prime}$.

When $G$ has trivial centre, which is the case considered in [1, there exists a unique $I$-canonical element, which is given by $\xi_{I}=\sum_{i \in I} H_{i}$, for each $I$. When $G$ has non-trivial centre, the $I$-canonical elements of $G$ were described in [5, 6].

Any harmonic map $\varphi: S^{2} \rightarrow G$ admits a normalized extended solution, that is, an extended solution $\Phi$ taking values in $U_{\xi}(G)$, off some discrete set, for some canonical element $\xi$. This is a consequence of the following generalization of Theorem 4.5 in [1].

Theorem 5. 4] Let $\Phi: S^{2} \backslash D \rightarrow U_{\xi}(G)$ be an extended solution. Take $\xi^{\prime} \in \mathfrak{I}^{\prime}(G)$ such that $\xi \preceq \xi^{\prime}$ and $\mathfrak{g}_{0}^{\xi}=\mathfrak{g}_{0}^{\xi^{\prime}}$. Then $\gamma^{-1}:=\mathcal{U}_{\xi, \xi-\xi^{\prime}}(\Phi)$ is a constant loop in $\Omega_{\text {alg }} G$ and $\gamma \Phi: S^{2} \backslash D \rightarrow U_{\xi^{\prime}}(G)$.

The uniton number of a normalized extended solution $\Phi: S^{2} \backslash D \rightarrow U_{\xi}(G)$ can be computed with respect to any irreducible $n$-dimensional representation $\rho: G \rightarrow \operatorname{End}(V)$ with highest weight $\omega^{*}$ and lowest weight $\varpi^{*}$ as follows []: $r_{\rho}(\xi):=\omega^{*}(\xi)-\varpi^{*}(\xi)$.

\section{HARMONIC SPHERES IN OUTER SYMMETRIC SPACES}

In the following sections we will establish our classification of harmonic maps from $S^{2}$ into compact outer symmetric spaces and establish a Weierstrass formula for such harmonic maps. These will allow us to produce some explicit examples of harmonic maps from two-spheres into outer symmetric spaces from meromorphic data.

As we have referred in Section 1, although any harmonic map from a two-sphere into an outer symmetric space $G / K$ admits a $T_{\sigma}$-invariant extended solution, not all $T_{\sigma}$-invariant extended solutions correspond to harmonic maps into $G / K$; by Proposition 6 and Theorem 10 below, they correspond to a harmonic map into some possibly different outer symmetric space $G / K^{\prime}$ (compare Theorem 20 with Theorem 23 for an example where this happens).

4.1. Symmetric $G$-spaces and Cartan embeddings. Let $N=G / K$ be a symmetric space, where $K$ is the isotropy subgroup at the base point $x_{0} \in N$, and let $\sigma: G \rightarrow G$ be the corresponding involution: we have $G_{0}^{\sigma} \subseteq K \subseteq G^{\sigma}$, where $G^{\sigma}$ is the subgroup fixed by $\sigma$ and $G_{0}^{\sigma}$ denotes its connected component of the identity. We assume that $N$ is a bottom space, i.e. $K=G^{\sigma}$. Let $\mathfrak{g}=\mathfrak{k}_{\sigma} \oplus \mathfrak{m}_{\sigma}$ be the \pm 1 -eigenspace decomposition associated to the involution $\sigma$, where $\mathfrak{k}_{\sigma}$ is the Lie algebra of $K$. Consider the (totally geodesic) Cartan embedding $\iota_{\sigma}: N \hookrightarrow G$ defined by $\iota_{\sigma}\left(g \cdot x_{0}\right)=g \sigma\left(g^{-1}\right)$. The image of the Cartan embedding is precisely the connected component $P_{e}^{\sigma}$ of $P^{\sigma}:=\left\{g \in G \mid \sigma(g)=g^{-1}\right\}$ containing the identity $e$ of the group $G$. Observe that, given $\xi \in \mathfrak{I}(G) \cap \mathfrak{k}_{\sigma}$, then $\exp (\pi \xi) \in P^{\sigma}$. We denote by $P_{\xi}^{\sigma}$ the connected component of $P^{\sigma}$ containing $\exp (\pi \xi)$.

Proposition 6. Given $\xi \in \mathfrak{I}(G) \cap \mathfrak{k}_{\sigma}$, we have the following.

a) $G$ acts transitively on $P_{\xi}^{\sigma}$ as follows: for $g \in G$ and $h \in P_{\xi}^{\sigma}$,

$$
g \cdot{ }_{\sigma} h=g h \sigma\left(g^{-1}\right) .
$$

b) $P_{\xi}^{\sigma}$ is a bottom symmetric $G$-space totally geodesically embedded in $G$ with involution

$$
\tau=\operatorname{Ad}(\exp \pi \xi) \circ \sigma .
$$

c) For any other $\xi^{\prime} \in \mathfrak{I}(G) \cap \mathfrak{k}_{\sigma}$ we have $\exp \left(\pi \xi^{\prime}\right) \in P^{\tau}$ and $P_{\xi^{\prime}}^{\tau}=\exp (\pi \xi) P_{\xi^{\prime}-\xi}^{\sigma}$. 
d) The \pm 1-eigenspace decomposition $\mathfrak{g}=\mathfrak{k}_{\tau} \oplus \mathfrak{m}_{\tau}$ associated to the symmetric $G$-space $P_{\xi}^{\sigma}$ at the fixed point $\exp (\pi \xi) \in P_{\xi}^{\sigma}$ is given by

$$
\begin{aligned}
\mathfrak{k}_{\tau}^{\mathbb{C}} & =\bigoplus \mathfrak{g}_{2 i}^{\xi} \cap \mathfrak{k}_{\sigma}^{\mathbb{C}} \oplus \bigoplus \mathfrak{g}_{2 i+1}^{\xi} \cap \mathfrak{m}_{\sigma}^{\mathbb{C}} \\
\mathfrak{m}_{\tau}^{\mathbb{C}} & =\bigoplus \mathfrak{g}_{2 i+1}^{\xi} \cap \mathfrak{k}_{\sigma}^{\mathbb{C}} \oplus \bigoplus \mathfrak{g}_{2 i}^{\xi} \cap \mathfrak{m}_{\sigma}^{\mathbb{C}}
\end{aligned}
$$

Proof. Take $h \in P^{\sigma}$. We have

$$
\sigma(g \cdot \sigma h)=\sigma\left(g h \sigma\left(g^{-1}\right)\right)=\sigma(g) h^{-1} g^{-1}=\left(g h \sigma\left(g^{-1}\right)\right)^{-1}=\left(g \cdot{ }_{\sigma} h\right)^{-1} .
$$

Then $g \cdot_{\sigma} h \in P^{\sigma}$ and we have a continuous action of $G$ on $P^{\sigma}$. Since $G$ is connected, this action induces an action of $G$ on each connected component of $P^{\sigma}$. Since $g \cdot_{\sigma} e=g \sigma\left(g^{-1}\right)=\iota_{\sigma}\left(g \cdot x_{0}\right)$ and $\iota_{\sigma}(N)=P_{e}^{\sigma}$, the action $\cdot{ }_{\sigma}$ of $G$ on $P_{e}^{\sigma}$ is transitive.

Take $\xi \in \mathfrak{I}(G) \cap \mathfrak{k}_{\sigma}$, so that $\sigma(\xi)=\xi$ and $\exp 2 \pi \xi=e$. Consider the involution $\tau$ defined by (4). If $g \in P^{\sigma}$, then

$$
\tau(\exp (\pi \xi) g)=\exp (\pi \xi) \sigma(\exp (\pi \xi) g) \exp (\pi \xi)=\sigma(g) \exp (\pi \xi)=(\exp (\pi \xi) g)^{-1},
$$

which means that $\exp (\pi \xi) g \in P^{\tau}$. Reciprocally, if $\exp (\pi \xi) g \in P^{\tau}$, one can check similarly that $g \in P^{\sigma}$. Hence $P^{\tau}=\exp (\pi \xi) P^{\sigma}$. In particular, by continuity, $P_{\xi^{\prime}}^{\tau}=\exp (\pi \xi) P_{\xi^{\prime}-\xi}^{\sigma}$ for any other $\xi^{\prime} \in \Im(G)$ with $\sigma\left(\xi^{\prime}\right)=\xi^{\prime}$.

Reversing the rules of $\sigma=\operatorname{Ad}(\exp \pi \xi) \circ \tau$ and $\tau$, we also have $P_{\xi}^{\sigma}=\exp (\pi \xi) P_{e}^{\tau}$. Since $G$ acts transitively on $P_{e}^{\tau}$, for each $h \in P_{\xi}^{\sigma}$ there exists $g \in G$ such that

$$
h=\exp (\pi \xi)\left(g \cdot_{\tau} e\right)=(\exp (\pi \xi) g) \cdot{ }_{\sigma} \exp (\pi \xi) .
$$

This shows that $G$ also acts transitively on $P_{\xi}^{\sigma}$. The isotropy subgroup at $\exp (\pi \xi)$ consists of those elements $g$ of $G$ satisfying $g \exp (\pi \xi) \sigma\left(g^{-1}\right)=\exp (\pi \xi)$, that is those elements $g$ of $G$ which are fixed by $\tau$ :

$$
\exp (\pi \xi) \sigma(g) \exp (\pi \xi)=g .
$$

Hence $P_{\xi}^{\sigma} \cong G / G^{\tau}$, which is a bottom symmetric $G$-space with involution $\tau$. Since $P_{e}^{\tau} \subset G$ totally geodesically and $P_{\xi}^{\sigma}$ is the image of $P_{e}^{\tau}$ under an isometry (left multiplication by $\exp \pi \xi$ ), then $P_{\xi}^{\sigma} \subset G$ totally geodesically.

Differentiating (77) at the identity we get $\mathfrak{k}_{\tau}=\{X \in \mathfrak{g} \mid X=\operatorname{Ad}(\exp \pi \xi) \circ \sigma(X)\}$. Taking account of the formula $\operatorname{Ad}(\exp (\pi \xi))=e^{\pi \operatorname{ad} \xi}$ and that $\sigma$ commutes with $\operatorname{ad} \xi$, we obtain (5); and (6) follows similarly.

4.1.1. Outer symmetric spaces. The existence of outer involutions of a simple Lie algebra $\mathfrak{g}$ depends on the existence of non-trivial involutions of the Dynkin diagram of $\mathfrak{g}^{\mathbb{C}}$ 2, 8, 12, 14. Fix a maximal abelian subalgebra $\mathfrak{t}$ of $\mathfrak{g}$ and a Weyl chamber $\mathcal{W}$ in $\mathfrak{t}$, which amounts to fix a system of positive simple roots $\Delta_{0}=\left\{\alpha_{1}, \ldots, \alpha_{r}\right\}$, where $r=\operatorname{rank}(\mathfrak{g})$. Let $\varrho$ be a non-trivial involution of the Dynkin diagram and $\sigma_{\varrho}$ the fundamental outer involution associated to $\varrho$ [2, 14]. The (local isometry classes of) outer symmetric spaces of compact type associated to involutions of the form $\sigma_{\varrho}$ are precisely

$$
S U(2 n) / S p(n), S U(2 n+1) / S O(2 n+1), E_{6} / F_{4} \text { and the real projective spaces } \mathbb{R} P^{2 n-1} .
$$

These spaces are called the fundamental outer symmetric spaces. The remaining classes of outer involutions are obtained as follows 1, 14.

Let $\mathfrak{g}=\mathfrak{k}_{\varrho} \oplus \mathfrak{m}_{\varrho}$ be the corresponding \pm 1 -eigenspace decomposition of $\mathfrak{g}$. As shown in Proposition 3.20 of 2], the Lie subalgebra $\mathfrak{k}_{\varrho}$ is simple and the orthogonal projection of $\Delta_{0}$ onto $\mathfrak{k}_{\varrho}, \pi_{\mathfrak{k}_{\varrho}}\left(\Delta_{0}\right)$, is a basis of positive simple roots of $\mathfrak{k}_{\varrho}$ associated to the maximal abelian subalgebra $\mathfrak{t}_{\mathfrak{k}}:=\mathfrak{t} \cap \mathfrak{k}_{\varrho}$. Consider the split $\mathfrak{t}=\mathfrak{t}_{\mathfrak{k}} \oplus \mathfrak{t}_{\mathfrak{m}_{\varrho}}$ with respect to $\mathfrak{g}=\mathfrak{k}_{\varrho} \oplus \mathfrak{m}_{\varrho}$. Set $s=r-k$, where $k=\operatorname{rank}\left(\mathfrak{k}_{\varrho}\right)$. We can label the basis $\Delta_{0}$ in order to get the following relations: $\varrho\left(\alpha_{j}\right)=\alpha_{j}$ for $1 \leq j \leq k-s$ and $\varrho\left(\alpha_{j}\right)=\alpha_{s+j}$ for $k-s+1 \leq j \leq k$. Let $\pi_{\mathfrak{k}_{\varrho}}$ be the orthogonal projection of $\mathfrak{t}$ onto $\mathfrak{t}_{\mathfrak{k}}$, that is $\pi_{\mathfrak{k}_{\varrho}}(H)=\frac{1}{2}\left(H+\sigma_{\varrho}(H)\right)$ for all $H \in \mathfrak{t}$. Set $\pi_{\mathfrak{k}_{e}}\left(\Delta_{0}\right)=\left\{\beta_{1}, \ldots, \beta_{k}\right\}$, with

$$
\beta_{j}=\left\{\begin{array}{cl}
\alpha_{j} & \text { for } 1 \leq j \leq k-s \\
\frac{1}{2}\left(\alpha_{j}+\alpha_{j+s}\right) & \text { for } k-s+1 \leq j \leq k
\end{array} .\right.
$$


This is a basis of $\mathrm{it}_{\mathfrak{k}_{e}}^{*}$ with dual basis $\left\{\zeta_{1}, \ldots, \zeta_{k}\right\}$ given by

$$
\zeta_{j}=\left\{\begin{array}{cl}
H_{j} & \text { for } 1 \leq j \leq k-s \\
H_{j}+H_{j+s} & \text { for } k-s+1 \leq j \leq k
\end{array} .\right.
$$

Theorem 7. 14] Let $\varrho$ be an involution of the Dynkin diagram of $\mathfrak{g}$. Let

$$
\omega=\sum_{j=1}^{k-s} n_{j} \beta_{j}+\sum_{j=k-s+1}^{k} n_{j}^{\prime} \beta_{j}
$$

be the highest root of $\mathfrak{k}_{\varrho}$ with respect to $\pi_{\mathfrak{k}}\left(\Delta_{0}\right)=\left\{\beta_{1}, \ldots, \beta_{k}\right\}$, defined as in (8). Given $i$ such that $n_{i}=1$ or 2 , define an involution $\sigma_{\varrho, i}$ by

$$
\sigma_{\varrho, i}=\operatorname{Ad}\left(\exp \pi \zeta_{i}\right) \circ \sigma_{\varrho} .
$$

Then any outer involution of $\mathfrak{g}$ is conjugate in $\mathfrak{A} \mathfrak{u}(\mathfrak{g})$, the group of automorphisms of $\mathfrak{g}$, to some $\sigma_{\varrho}$ or $\sigma_{\varrho, i}$. In particular, there are at most $k-s+1$ conjugacy classes of outer involutions.

The list of all (local isometry classes of) irreducible outer symmetric spaces of compact type is shown in Table 1 (cf. [2, 8, 12, ).

\begin{tabular}{|c|c|c|c|c|}
\hline$G / K$ & $\operatorname{rank}(G)$ & $\operatorname{rank}(K)$ & $\operatorname{rank}(G / K)$ & $\operatorname{dim}(G / K)$ \\
\hline$S U(2 n) / S O(2 n)$ & $2 n-1$ & $n$ & $2 n-1$ & $(2 n-1)(n+1)$ \\
\hline$S U(2 n+1) / S O(2 n+1)$ & $2 n$ & $n$ & $2 n$ & $n(2 n+3)$ \\
\hline$S U(2 n) / S p(n)$ & $2 n-1$ & $n$ & $n-1$ & $(n-1)(2 n+1)$ \\
\hline$G_{p}\left(\mathbb{R}^{2 n}\right)(p$ odd $\leq n)$ & $n$ & $n-1$ & $p$ & $p(2 n-p)$ \\
\hline$E_{6} / S p(4)$ & 6 & 4 & 6 & 42 \\
\hline$E_{6} / F_{4}$ & 6 & 4 & 2 & 26 \\
\hline
\end{tabular}

TABLE 1. Irreducible outer symmetric spaces.

Given an outer involution $\sigma$ of the form $\sigma_{\varrho, i}$ or $\sigma_{\varrho}$ and its \pm -eigenspace decomposition $\mathfrak{g}=\mathfrak{k}_{\sigma} \oplus \mathfrak{m}_{\sigma}$, set $\mathfrak{t}_{\mathfrak{k}_{\sigma}}=\mathfrak{t} \cap \mathfrak{k}_{\sigma}$, which is a maximal abelian subalgebra of $\mathfrak{k}_{\sigma}$. Following [8], a non-empty intersection of $\mathfrak{t}_{\mathfrak{k}_{\sigma}}$ with a Weyl chamber in $\mathfrak{t}$ is called a compartment. Each compartment lies in a Weyl chamber in $\mathfrak{t}_{\mathfrak{k}_{\sigma}}$ and the Weyl chambers in $\mathfrak{t}_{\mathfrak{k}_{\sigma}}$ can be decomposed into the same number of compartments [].

The intersection of the integer lattice $\mathfrak{I}(G)$ with the Weyl chamber $\mathcal{W}$ in $\mathfrak{t}$, which we have denoted by $\mathfrak{I}^{\prime}(G)$, is described in terms of the dual basis $\left\{H_{1}, \ldots, H_{r}\right\} \subset \mathfrak{t}$, with $r=\operatorname{rank}(\mathfrak{g})$, by

$$
\mathfrak{I}^{\prime}(G)=\left\{\sum_{i=1}^{r} n_{i} H_{i} \in \mathfrak{I}(G) \mid n_{i} \in \mathbb{N}_{0} \text { for all } i\right\} .
$$

When $\sigma$ is a fundamental outer involution $\sigma_{\varrho}$, the compartment $\mathcal{W} \cap \mathfrak{t}_{\mathfrak{k}}$ is itself a Weyl chamber in $\mathfrak{t}_{\mathfrak{k}_{\varrho}}$. Then, the intersection of the integer lattice $\mathfrak{I}\left(G^{\sigma_{\varrho}}\right)$ with the Weyl chamber $\mathcal{W} \cap \mathfrak{t}_{\mathfrak{k}_{\varrho}}$, is given by

$$
\mathfrak{I}^{\prime}\left(G^{\sigma_{\varrho}}\right)=\left\{\sum_{i=1}^{k} n_{i} \zeta_{i} \in \mathfrak{I}(G) \mid n_{i} \in \mathbb{N}_{0} \text { for all } i\right\}=\mathfrak{I}^{\prime}(G) \cap \mathfrak{t}_{\mathfrak{k}_{\varrho}} .
$$

4.1.2. Cartan embeddings of fundamental outer symmetric spaces. Next we describe those elements $\xi$ of $\mathfrak{I}^{\prime}\left(G^{\sigma_{\varrho}}\right)$ for which the connected component $P_{\xi}^{\sigma_{\varrho}}$ of $P^{\sigma_{\varrho}}$ containing $\exp (\pi \xi)$ can be identified with the fundamental outer symmetric $G$-space associated to $\varrho$. Start by considering the following $\sigma_{\varrho}$-invariant subsets of the root system $\Delta \subset$ it* of $\mathfrak{g}:$

$$
\Delta\left(\mathfrak{k}_{\varrho}\right)=\left\{\alpha \in \Delta \mid \mathfrak{g}_{\alpha} \subset \mathfrak{k}_{\varrho}^{\mathbb{C}}\right\}, \Delta\left(\mathfrak{m}_{\varrho}\right)=\left\{\alpha \in \Delta \mid \mathfrak{g}_{\alpha} \subset \mathfrak{m}_{\varrho}^{\mathbb{C}}\right\}, \Delta_{\varrho}=\Delta \backslash\left(\Delta\left(\mathfrak{k}_{\varrho}\right) \cup \Delta\left(\mathfrak{m}_{\varrho}\right)\right) .
$$


Then

$$
\mathfrak{k}_{\varrho}^{\mathbb{C}}=\mathfrak{t}_{\mathfrak{k}_{\varrho}}^{\mathbb{C}} \oplus \pi_{\mathfrak{k}_{\varrho}}\left(\mathfrak{r}_{\varrho}\right) \oplus \bigoplus_{\alpha \in \Delta\left(\mathfrak{k}_{\varrho}\right)} \mathfrak{g}_{\alpha}, \quad \mathfrak{m}_{\varrho}^{\mathbb{C}}=\mathfrak{t}_{\mathfrak{m}_{\varrho}}^{\mathbb{C}} \oplus \pi_{\mathfrak{m}_{\varrho}}\left(\mathfrak{r}_{\varrho}\right) \oplus \bigoplus_{\alpha \in \Delta\left(\mathfrak{m}_{\varrho}\right)} \mathfrak{g}_{\alpha},
$$

where $\mathfrak{r}_{\varrho}=\bigoplus_{\alpha \in \Delta_{\varrho}} \mathfrak{g}_{\alpha}$. Since the involution $\varrho$ acts on $\Delta_{\varrho}$ as a permutation without fixed points, we can fix some subset $\Delta_{\varrho}^{\prime}$ so that $\Delta_{\varrho}$ is the disjoint union of $\Delta_{\varrho}^{\prime}$ with $\varrho\left(\Delta_{\varrho}^{\prime}\right)$ :

$$
\Delta_{\varrho}=\Delta_{\varrho}^{\prime} \sqcup \varrho\left(\Delta_{\varrho}^{\prime}\right) .
$$

For each $\alpha \in \Delta_{\varrho}^{\prime}, \sigma_{\varrho}$ restricts to an involution in the subspace $\mathfrak{g}_{\alpha} \oplus \mathfrak{g}_{\varrho(\alpha)} \subset \mathfrak{r}_{\varrho}$. Hence we have the following.

Lemma 8. The orthogonal projections of $\mathfrak{r}_{\varrho}$ onto $\mathfrak{k}_{\varrho}^{\mathbb{C}}$ and $\mathfrak{m}_{\varrho}^{\mathbb{C}}$ are given by

$$
\pi_{\mathfrak{k}_{\varrho}}\left(\mathfrak{r}_{\varrho}\right)=\bigoplus_{\alpha \in \Delta_{\varrho}^{\prime}} \mathfrak{k}_{\varrho}^{\mathbb{C}} \cap\left(\mathfrak{g}_{\alpha} \oplus \mathfrak{g}_{\varrho(\alpha)}\right), \pi_{\mathfrak{m}_{\varrho}}\left(\mathfrak{r}_{\varrho}\right)=\bigoplus_{\alpha \in \Delta_{\varrho}^{\prime}} \mathfrak{m}_{\varrho}^{\mathbb{C}} \cap\left(\mathfrak{g}_{\alpha} \oplus \mathfrak{g}_{\varrho(\alpha)}\right),
$$

and, for each $\alpha \in \Delta_{\varrho}^{\prime}$,

$$
\mathfrak{k}_{\varrho}^{\mathbb{C}} \cap\left(\mathfrak{g}_{\alpha} \oplus \mathfrak{g}_{\sigma_{\varrho}(\alpha)}\right)=\left\{X_{\alpha}+\sigma_{\varrho}\left(X_{\alpha}\right) \mid X_{\alpha} \in \mathfrak{g}_{\alpha}\right\}, \mathfrak{m}_{\varrho}^{\mathbb{C}} \cap\left(\mathfrak{g}_{\alpha} \oplus \mathfrak{g}_{\sigma(\alpha)}\right)=\left\{X_{\alpha}-\sigma_{\varrho}\left(X_{\alpha}\right) \mid X_{\alpha} \in \mathfrak{g}_{\alpha}\right\} .
$$

In particular, $\operatorname{dim} \mathfrak{r}_{\varrho}=2 \operatorname{dim} \pi_{\mathfrak{k}_{\varrho}}\left(\mathfrak{r}_{\varrho}\right)=2 \operatorname{dim} \pi_{\mathfrak{m}_{\varrho}}\left(\mathfrak{r}_{\varrho}\right)$.

Proposition 9. Consider the dual basis $\left\{\zeta_{1}, \ldots, \zeta_{k}\right\}$ defined by (9). Given $\xi \in \mathfrak{I}^{\prime}\left(G^{\sigma_{\varrho}}\right)$ with $\xi=\sum_{i=1}^{k} n_{i} \zeta_{i}$ and $n_{i} \geq 0$, then $P_{\xi}^{\sigma_{e}}$ is a fundamental outer symmetric space with involution (conjugated to) $\sigma_{\varrho}$ if and only if $n_{i}$ is even for each $1 \leq i \leq k-s$.

Proof. There is only one class of outer symmetric $S U(2 n+1)$-spaces and, in this case, the involution $\varrho$ does not fix any simple root, that is $k-s=0$. Hence the result trivially holds for $N=S U(2 n+1) / S O(2 n+1)$.

Next we consider the remaining fundamental outer symmetric spaces, which are precisely the symmetric spaces of rank-split type [8], those satisfying $\Delta\left(\mathfrak{m}_{\varrho}\right)=\emptyset$. For such symmetric spaces, the reductive symmetric term $\mathfrak{m}_{\varrho}$ satisfies $\mathfrak{m}_{\varrho}=\mathfrak{t}_{\mathfrak{m}_{\varrho}} \oplus \pi_{\mathfrak{m}_{\varrho}}\left(\mathfrak{r}_{\varrho}\right)$. On the other hand, in view of (ㅌ), we have, for $\tau=\operatorname{Ad}(\exp \pi \xi) \circ \sigma_{\varrho}$,

$$
\begin{aligned}
\mathfrak{m}_{\tau}^{\mathbb{C}} & =\bigoplus \mathfrak{g}_{2 i+1}^{\xi} \cap \mathfrak{k}_{\varrho}^{\mathbb{C}} \oplus \bigoplus \mathfrak{g}_{2 i}^{\xi} \cap \mathfrak{m}_{\varrho}^{\mathbb{C}} \\
& =\mathfrak{t}_{\mathfrak{m}_{\varrho}}^{\mathbb{C}} \oplus \underset{\alpha \in \Delta\left(\mathfrak{k}_{e}\right) \cap \Delta_{\xi}^{-}}{\bigoplus} \mathfrak{g}_{\alpha} \oplus \bigoplus_{\alpha \in \Delta_{\varrho}^{\prime} \cap \Delta_{\xi}^{-}} \mathfrak{k}_{\varrho}^{\mathbb{C}} \cap\left(\mathfrak{g}_{\alpha} \oplus \mathfrak{g}_{\varrho(\alpha)}\right) \oplus \underset{\alpha \in \Delta_{\varrho}^{\prime} \cap \Delta_{\xi}^{+}}{\bigoplus} \mathfrak{m}_{\varrho}^{\mathbb{C}} \cap\left(\mathfrak{g}_{\alpha} \oplus \mathfrak{g}_{\varrho(\alpha)}\right),
\end{aligned}
$$

where $\Delta_{\xi}^{+}:=\{\alpha \in \Delta \mid \alpha(\xi)$ i is even $\}$ and $\Delta_{\xi}^{-}:=\{\alpha \in \Delta \mid \alpha(\xi)$ i is odd $\}$. Taking into account Lemma 8 , from this we see that $\operatorname{dim} \mathfrak{m}_{\tau}=\operatorname{dim} \mathfrak{m}_{\varrho}$ (which means, by Table 1 , that $P_{\xi}^{\sigma_{\varrho}}$ is a fundamental outer symmetric space with involution conjugated to $\sigma_{\varrho}$ ) if and only if

$$
\bigoplus_{\alpha \in \Delta\left(\mathfrak{k}_{e}\right) \cap \Delta_{\xi}^{-}} \mathfrak{g}_{\alpha}=\{0\},
$$

which holds if and only if $\xi=\sum_{i=1}^{k} n_{i} \zeta_{i}$ with $n_{i}$ even for each $1 \leq i \leq k-s$.

4.2. Harmonic spheres in symmetric $G$-spaces. Given an involution $\sigma$ on $G$, define an involution $T_{\sigma}$ on $\Omega G$ by $T_{\sigma}(\gamma)(\lambda)=\sigma\left(\gamma(-\lambda) \gamma(-1)^{-1}\right)$. Let $\Omega^{\sigma} G$ be the fixed set of $T_{\sigma}$.

Theorem 10. [8, 11] Given $\xi \in \mathfrak{I}(G) \cap \mathfrak{k}_{\sigma}$, any harmonic map $\varphi: S^{2} \rightarrow P_{\xi}^{\sigma} \subset G$ admits an $T_{\sigma}$-invariant extended solution $\Phi: S^{2} \rightarrow \Omega^{\sigma} G$. Conversely, given an $T_{\sigma}$-invariant extended solution $\Phi$, the smooth map $\varphi=\Phi_{-1}$ from $S^{2}$ is harmonic and takes values in some connected component of $P^{\sigma}$.

Proposition 11. [8] Given $\Phi \in U_{\xi}^{\sigma}(G):=U_{\xi}(G) \cap \Omega^{\sigma} G$, with $\xi \in \mathfrak{I}(G) \cap \mathfrak{k}_{\sigma}$, set $\gamma=u_{\xi} \circ \Phi$. Then $\gamma$ takes values in $K$. By continuity, $\Phi_{-1}$ and $\gamma(-1)$ take values in the same connected component of $P^{\sigma}$.

Hence, together with Theorems 1 and 10, this implies the following. 
Theorem 12. Any harmonic map $\varphi$ from $S^{2}$ into a connected component of $P^{\sigma}$ admits an extended solution $\Phi: S^{2} \backslash D \rightarrow U_{\xi}^{\sigma}(G):=U_{\xi}(G) \cap \Omega^{\sigma} G$, for some $\xi \in \mathfrak{I}^{\prime}(G) \cap \mathfrak{k}_{\sigma}$ and some discrete subset $D$. If $\sigma=\sigma_{\varrho}$ is the fundamental outer involution, then $\varphi=\Phi_{-1}$ takes values in $P_{\xi}^{\sigma_{Q}}$.

Proof. By Proposition 11 $\Phi$ and $\gamma:=u_{\xi} \circ \Phi$ take values in the same connected component of $P^{\sigma}$ when evaluated at $\lambda=-1$. Since $\gamma: S^{1} \rightarrow G^{\sigma}$ is a homomorphism, $\gamma$ is in the $G^{\sigma}$-conjugacy class of $\gamma_{\xi^{\prime}}$ for some $\xi^{\prime} \in \mathfrak{I}^{\prime}\left(G^{\sigma}\right)$, where $G^{\sigma}$ is the subgroup of $G$ fixed by $\sigma$. Consequently, $\gamma(-1)=g \gamma_{\xi^{\prime}}(-1) g^{-1}=g{ }_{\sigma} \gamma_{\xi^{\prime}}(-1)$, for some $g \in G^{\sigma}$, which means that $\gamma(-1)$ takes values in the connected component $P_{\xi^{\prime}}^{\sigma}$. On the other hand, $\gamma$ is in the $G$ conjugacy class of $\gamma_{\xi}$, with $\xi \in \mathfrak{I}^{\prime}(G) \cap \mathfrak{k}_{\sigma}$. If $\sigma$ is the fundamental outer involution $\sigma_{\varrho}$, then $\mathfrak{I}^{\prime}\left(G^{\sigma}\right)=\mathfrak{I}^{\prime}(G) \cap \mathfrak{k}_{\sigma}$; and we must have $\xi=\xi^{\prime}$.

Remark 1. If $\sigma$ is not a fundamental outer involution, each Weyl chamber $\mathcal{W}_{\sigma}$ in $\mathfrak{t}_{\mathfrak{k}_{\sigma}}$ can be decomposed into more than one compartment: $\mathcal{W}_{\sigma}=C_{1} \sqcup \ldots \sqcup C_{l}$, where $C_{1}=\mathcal{W} \cap \mathfrak{t}_{\mathfrak{k}_{\sigma}}$ and the remaining compartments are conjugate to $C_{1}$ under $G$ [8], that is, there exists $g_{i} \in G$ satisfying $C_{i}=\operatorname{Ad}\left(g_{i}\right)\left(C_{1}\right)$ for each $i$. Hence, if we have an extended solution $\Phi: S^{2} \backslash D \rightarrow U_{\xi}^{\sigma}(G)$ with $\xi \in \mathfrak{I}^{\prime}(G) \cap \mathfrak{k}_{\sigma} \subset C_{1}$, the corresponding harmonic map $\Phi_{-1}$ takes values in one of the connected components $P_{g_{i} \xi g_{i}^{-1}}^{\sigma}$.

4.2.1. $\varrho$-canonical elements. Let $I$ be a subset of $\{1, \ldots, k\}$, with $k=\operatorname{rank}\left(\mathfrak{k}_{\varrho}\right)$, and set

$$
\mathfrak{C}_{I}^{\varrho}=\left\{\sum_{i=1}^{k} n_{i} \zeta_{i} \mid n_{i} \geq 0, n_{j}=0 \text { iff } j \notin I\right\} .
$$

Let $\xi \in \mathfrak{I}^{\prime}\left(G^{\sigma_{\varrho}}\right) \cap \mathfrak{C}_{I}^{\varrho}$. We say that $\zeta$ is a $\varrho$-canonical element of $G$ (with respect to the choice of $\mathcal{W}$ ) if $\zeta$ is a maximal element of $\left(\mathfrak{I}^{\prime}\left(G^{\sigma_{\varrho}}\right) \cap \mathfrak{C}_{I}^{\varrho}, \preceq\right)$, that is: if $\zeta \preceq \zeta^{\prime}$ and $\zeta^{\prime} \in \mathfrak{I}^{\prime}\left(G^{\sigma_{\varrho}}\right) \cap \mathfrak{C}_{I}^{\varrho}$ then $\zeta=\zeta^{\prime}$.

Remark 2. When $G$ has trivial centre, the duals $\zeta_{1}, \ldots, \zeta_{k}$ belong to the integer lattice. Then, for each $I$ there exists a unique $\varrho$-canonical element, which is given by $\zeta_{I}=\sum_{i \in I} \zeta_{i}$. In this case, our definition of $\varrho$-canonical element coincides with that of $S$-canonical element in [8].

Now, consider a fundamental outer involution $\sigma_{\varrho}$ and let $N$ be an associated outer symmetric $G$-space, that is, $N$ corresponds to an involution of $G$ of the form $\sigma_{\varrho}$ or $\sigma_{\varrho, i}$, with $\zeta_{i}$ in the conditions of Theorem 7 . If $G$ has trivial centre, we certainly have $\zeta_{i} \in \mathfrak{I}^{\prime}\left(G^{\sigma_{\varrho}}\right)$. As a matter of fact, as we will see later, in most cases we have $\zeta_{i} \in \mathfrak{I}^{\prime}\left(G^{\sigma_{\varrho}}\right)$, whether $G$ has trivial centre or not, with essentially one exception: for $G=S U(2 n)$ and $N=S U(2 n) / S O(2 n)$. So, we will treat this case separately and assume henceforth that $\zeta_{i} \in \mathfrak{I}^{\prime}\left(G^{\sigma_{\varrho}}\right)$.

Remark 3. Consider the Dynkin diagram of $\mathfrak{e}_{6}$ :

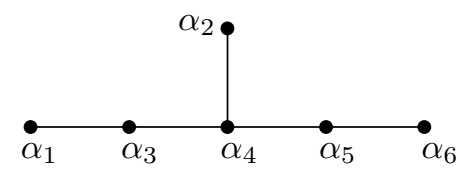

This admits a unique nontrivial involution $\varrho$. Let $\left\{H_{1}, \ldots, H_{6}\right\}$ be the dual basis of $\Delta_{0}=\left\{\alpha_{1}, \ldots, \alpha_{6}\right\}$. The semi-fundamental basis $\pi_{\mathfrak{k}_{e}}\left(\Delta_{0}\right)=\left\{\beta_{1}, \beta_{2}, \beta_{3}, \beta_{4}\right\}$ is given by $\beta_{1}=\alpha_{2}, \beta_{2}=\alpha_{4}, \beta_{3}=\frac{\alpha_{1}+\alpha_{6}}{2}$ and $\beta_{4}=\frac{\alpha_{3}+\alpha_{5}}{2}$, whereas the dual basis is given by $\zeta_{1}=H_{2}, \zeta_{2}=H_{4}, \zeta_{3}=H_{1}+H_{6}$ and $\zeta_{4}=H_{3}+H_{5}$. Taking account that the elements $H_{i}$ are related with the duals $\eta_{i}$ of the fundamental weights by

$$
\left[H_{i}\right]=\left[\begin{array}{cccccc}
4 / 3 & 1 & 5 / 3 & 2 & 4 / 3 & 2 / 3 \\
1 & 2 & 2 & 3 & 2 & 1 \\
5 / 3 & 2 & 10 / 3 & 4 & 8 / 3 & 4 / 3 \\
2 & 3 & 4 & 6 & 4 & 2 \\
4 / 3 & 2 & 8 / 3 & 4 & 10 / 3 & 5 / 3 \\
2 / 3 & 1 & 4 / 3 & 2 & 5 / 3 & 4 / 3
\end{array}\right]\left[\eta_{i}\right],
$$


we see that the elements $\zeta_{i}$ are in the integer lattice $\mathfrak{I}^{\prime}\left(\tilde{E}_{6}\right) \subset \mathfrak{I}^{\prime}\left(E_{6}\right)$, where $\tilde{E}_{6}$ is the compact simply connected Lie group with Lie algebra $\mathfrak{e}_{6}$, which has centre $\mathbb{Z}_{3}$, and $E_{6}$ is the adjoint group $\tilde{E}_{6} / \mathbb{Z}_{3}$.

Taking into account Proposition 6, we can identify $N$ with the connected component $P_{\zeta_{i}}^{\sigma_{\varrho}}=\exp \left(\pi \zeta_{i}\right) P_{e}^{\sigma_{\varrho, i}}$, which is a totally geodesic submanifold of $G$, via

$$
g \cdot x_{0} \in N \mapsto \exp \left(\pi \zeta_{i}\right) g \sigma_{\varrho, i}\left(g^{-1}\right) \in P_{\zeta_{i}}^{\sigma_{\varrho}} .
$$

By Theorem 12, each harmonic map $\varphi: S^{2} \rightarrow N \cong P_{\zeta_{i}}^{\sigma_{Q}}$ admits a $T_{\sigma_{e}}$-invariant extended solution with values, off a discrete set, in some unstable manifold $U_{\xi}(G)$, with $\xi \in \mathfrak{I}^{\prime}\left(G^{\sigma_{\varrho}}\right) \cap \mathfrak{C}_{I}^{\varrho}$. By Theorem [5, this extended solution can be multiplied on the left by a constant loop in order to get a normalized extended solution with values in some unstable manifold $U_{\zeta}(G)$ for some $\varrho$-canonical element $\zeta$. Hence, if $G$ has trivial centre, the Bruhat decomposition of $\Omega_{\mathrm{alg}} G$ gives rise to $2^{k}$ classes of harmonic maps into $P^{\sigma_{\varrho}}$, that is $2^{k}$ classes of harmonic maps into all outer symmetric $G$-spaces.

However, the normalization procedure given by Theorem 5 does not preserve $T_{\sigma_{e}}$-invariance, and consequently, as we will see next, normalized extended solutions with values in the same unstable manifold $U_{\zeta}(G)$, for some $\varrho$-canonical element $\zeta$, correspond in general to harmonic maps into different outer symmetric $G$-spaces. Hence the classification of harmonic two-spheres into outer symmetric $G$-spaces in terms of $\varrho$-canonical elements is manifestly unsatisfactory since it does not distinguishes the underlying symmetric space. In the following sections we overcome this weakness by establishing a classification of all such harmonic maps in terms of pairs $(\zeta, \sigma)$, where $\zeta$ is a $\varrho$-canonical element and $\sigma$ an outer involution of $G$.

4.2.2. Normalization of $T_{\sigma}$-invariant extended solutions. Let $\sigma$ be an outer involution of $G$. The fibre bundle morphisms $\mathcal{U}_{\xi, \xi^{\prime}}$ preserve $T_{\sigma^{-} \text {-invariance: }}$

Proposition 13. If $\xi \preceq \xi^{\prime}$ and $\xi, \xi^{\prime} \in \mathfrak{I}^{\prime}(G) \cap \mathfrak{k}_{\sigma}$, then $\mathcal{U}_{\xi, \xi^{\prime}}\left(U_{\xi}^{\sigma}(G)\right) \subset U_{\xi^{\prime}}^{\sigma}(G)$.

Proof. For $\Phi \in U_{\xi}^{\sigma}(G)$, write $\Phi=\Psi \cdot \gamma_{\xi}$ for some $\Psi \in \Lambda_{\text {alg }}^{+} G^{\mathbb{C}}$. If $\Phi$ is $T_{\sigma}$-invariant we have $\Psi(\lambda) \cdot \gamma_{\xi}=$ $\sigma(\Psi(-\lambda)) \cdot \gamma_{\xi}$. Consequently, we also have $\Psi(\lambda) \cdot \gamma_{\xi^{\prime}}=\sigma(\Psi(-\lambda)) \cdot \gamma_{\xi^{\prime}}$, which means in turn that $\mathcal{U}_{\xi, \xi^{\prime}}(\Phi)=\Psi \cdot \gamma_{\xi}^{\prime}$ is $T_{\sigma}$-invariant.

Hence, if $\Phi: S^{2} \backslash D \rightarrow U_{\xi}^{\sigma}(G)$ is an extended solution and $\xi \preceq \xi^{\prime}$, with $\xi, \xi^{\prime} \in \mathfrak{I}^{\prime}(G) \cap \mathfrak{k}_{e}$, by Theorem 5 and Proposition 13 we know that $\gamma^{-1}:=\mathcal{U}_{\xi, \xi-\xi^{\prime}}(\Phi)$ is a constant $T_{\sigma}$-invariant loop if $\mathfrak{g}_{0}^{\xi}=\mathfrak{g}_{0}^{\xi^{\prime}}$. However, in general, the product $\gamma \Phi$ is not $T_{\sigma}$-invariant.

Lemma 14. Assume that $\gamma^{-1}, \Phi \in \Omega^{\sigma} G$ and $\gamma(-1) \in P_{\xi}^{\sigma}$ for some $\xi \in \mathfrak{I}(G) \cap \mathfrak{k}_{\sigma}$. Take $h \in G$ such that $\gamma(-1)=h^{-1} \cdot_{\sigma} \exp (\pi \xi)$. Then $h \gamma \Phi h^{-1} \in \Omega^{\tau} G$, with $\tau=\operatorname{Ad}(\exp \pi \xi) \circ \sigma$.

Proof. Since $\gamma^{-1}, \Phi \in \Omega^{\sigma} G$, a simple computation shows that $T_{\sigma}(\gamma \Phi)=\gamma(-1)^{-1} \gamma \Phi \gamma(-1)$. Since $\gamma(-1) \in P_{\xi}^{\sigma}$, there exists $h \in G$ such that $\gamma(-1)=h^{-1} \cdot{ }_{\sigma} \exp (\pi \xi)=h^{-1} \exp (\pi \xi) \sigma(h)$. One can check now that $T_{\tau}\left(h \gamma \Phi h^{-1}\right)=$ $h \gamma \Phi h^{-1}$.

Proposition 15. Take $\xi, \xi^{\prime} \in \mathfrak{I}^{\prime}(G) \cap \mathfrak{k}_{\sigma}$ such that $\xi \preceq \xi^{\prime}$. Let $\Phi: S^{2} \backslash D \rightarrow U_{\xi}^{\sigma}(G)$ be a $T_{\sigma}$-invariant extended solution. If $\gamma^{-1}:=\mathcal{U}_{\xi, \xi-\xi^{\prime}}(\Phi)$ is a constant loop, there exists $h \in G$ such that $\tilde{\Phi}:=h \gamma \Phi h^{-1}$ takes values in $U_{\xi^{\prime}}^{\tau}(G)$, with $\tau=\operatorname{Ad}\left(\exp \pi\left(\xi-\xi^{\prime}\right)\right) \circ \sigma$.

Additionally, if $\sigma$ is the fundamental outer involution $\sigma_{\varrho}$, the harmonic map $\Phi_{-1}$ takes values in $P_{\xi}^{\sigma}$ and $\tilde{\Phi}_{-1}$ takes values in $P_{\xi^{\prime}}^{\tau}$, which implies that $\Phi_{-1}$ is given, up to isometry, by

$$
\exp \left(\pi\left(\xi-\xi^{\prime}\right)\right) \tilde{\Phi}_{-1}: S^{2} \rightarrow P_{\xi}^{\sigma}
$$

Proof. Assume that $\gamma^{-1}:=\mathcal{U}_{\xi, \xi-\xi^{\prime}}(\Phi)=\Psi \cdot \gamma_{\xi-\xi^{\prime}}$ is a constant loop. We can write $\Psi \gamma_{\xi-\xi^{\prime}}=\gamma^{-1} b$ for some $b: S^{2} \backslash D \rightarrow \Lambda_{\text {alg }}^{+} G$. Then

$$
\Phi=\Psi \cdot \gamma_{\xi}=\Psi \cdot \gamma_{\xi-\xi^{\prime}} \gamma_{\xi^{\prime}}=\gamma^{-1} b \cdot \gamma_{\xi^{\prime}}
$$


which implies that $\gamma \Phi$ takes values in $U_{\xi^{\prime}}(G)$. On the other hand, since $\gamma^{-1}$ is $T_{\sigma^{-}}$-invariant (by Proposition 13), $\gamma(-1) \in P^{\sigma}$.

Take $\eta \in \mathfrak{I}^{\prime}\left(G^{\sigma}\right)$ and $h \in G$ such that $\gamma(-1) \in P_{\eta}^{\sigma}$ and $\gamma(-1)=h^{-1} \cdot{ }_{\sigma} \exp \pi \eta$. From Lemma 14, we see that $\tilde{\Phi}:=h \gamma \Phi h^{-1}$ is $T_{\tau}$-invariant. Hence $\tilde{\Phi}$ takes values in $U_{\xi^{\prime}}^{\tau}(G)$. Since $\gamma$ is constant, $\tilde{\Phi}$ is an extended solution.

If $\sigma=\sigma_{\varrho}$, then $\mathfrak{I}^{\prime}\left(G^{\sigma_{\varrho}}\right)=\mathfrak{I}^{\prime}(G) \cap \mathfrak{k}_{\sigma_{\varrho}}$, which implies that $\eta=\xi-\xi^{\prime}$. The element $h \in G$ is such that

$$
\gamma(-1)=h^{-1} \exp \left(\pi\left(\xi-\xi^{\prime}\right)\right) \sigma_{\varrho}(h) .
$$

On the other hand, since, by Theorem 12, $\Phi_{-1}$ takes values in $P_{\xi}^{\sigma_{\varrho}}$, we also have $\Phi_{-1}=g \exp (\pi \xi) \sigma_{\varrho}\left(g^{-1}\right)$ for some lift $g: S^{2} \rightarrow G$. Hence

$$
\begin{aligned}
\tilde{\Phi}_{-1} & =h \gamma(-1) \Phi_{-1} h^{-1}=\exp \left(\pi\left(\xi-\xi^{\prime}\right)\right) \sigma_{\varrho}(h) g \exp (\pi \xi) \sigma_{\varrho}\left(\sigma_{\varrho}(h) g\right)^{-1} \\
& =\exp \left(\pi\left(\xi-\xi^{\prime}\right)\right)\left(\sigma_{\varrho}(h) g \cdot_{\sigma_{\varrho}} \exp \pi \xi\right)
\end{aligned}
$$

Hence, in view of Proposition [6, $\tilde{\Phi}_{-1}$ takes values in $P_{\xi^{\prime}}^{\tau}=\exp \left(\pi\left(\xi-\xi^{\prime}\right)\right) P_{\xi}^{\sigma}$.

Under some conditions on $\xi \preceq \xi^{\prime}$, the morphism $\mathcal{U}_{\xi, \xi-\xi^{\prime}}(\Phi)$ is always a constant loop.

Proposition 16. Take $\xi, \xi^{\prime} \in \mathfrak{I}^{\prime}(G) \cap \mathfrak{k}_{\sigma}$ such that $\xi \preceq \xi^{\prime}$. Assume that

$$
\mathfrak{g}_{2 i}^{\xi} \cap \mathfrak{m}_{\sigma}^{\mathbb{C}} \subset \bigoplus_{0 \leq j<2 i} \mathfrak{g}_{j}^{\xi-\xi^{\prime}}, \quad \mathfrak{g}_{2 i-1}^{\xi} \cap \mathfrak{k}_{\sigma}^{\mathbb{C}} \subset \bigoplus_{0 \leq j<2 i-1} \mathfrak{g}_{j}^{\xi-\xi^{\prime}}
$$

for all $i>0$. Then, $\mathcal{U}_{\xi, \xi-\xi^{\prime}}: U_{\xi}^{\sigma}(G) \rightarrow U_{\xi-\xi^{\prime}}^{\sigma}(G)$ transforms $T_{\sigma}$-invariant extended solutions in constant loops.

Proof. Given an extended solution $\Phi: S^{2} \backslash D \rightarrow U_{\xi}^{\sigma}(G)$, choose $\Psi: S^{2} \backslash D \rightarrow \Lambda_{\text {alg }}^{+} G^{\mathbb{C}}$ such that $\Phi=\Psi \cdot \gamma_{\xi}$ and $T_{\sigma}(\Psi)=\Psi$. Differentiating this we see that

$$
\operatorname{Im} \Psi^{-1} \Psi_{z} \subset \bigoplus_{i \geq 0} \lambda^{2 i} \mathfrak{k}_{\sigma}^{\mathbb{C}} \oplus \bigoplus_{i \geq 0} \lambda^{2 i+1} \mathfrak{m}_{\sigma}^{\mathbb{C}}
$$

Write $\Psi^{-1} \Psi_{z}=\sum_{r \geq 0} \lambda^{r} X_{r}^{\prime}$. Since $\xi \preceq \xi-\xi^{\prime}$, by Proposition 3 and Proposition $13, \mathcal{U}_{\xi, \xi-\xi^{\prime}}(\Phi)$ is an extended solution with values in $U_{\xi-\xi^{\prime}}^{\sigma}(G)$. Hence, taking into account Lemma 2 in order to prove that $\mathcal{U}_{\xi, \xi-\xi^{\prime}}(\Phi)$ is constant we only have to check that the component of $X_{r}^{\prime}$ over $\mathfrak{g}_{r+1}^{\xi-\xi^{\prime}}$ vanishes for all $r \geq 0$.

From (11) and (15) we see that, for $r=2 i, X_{2 i}^{\prime}$ takes values in $\bigoplus_{j \leq 2 i+1} \mathfrak{g}_{j}^{\xi} \cap \mathfrak{k}_{\sigma}^{\mathbb{C}}$. But, since $\xi \preceq \xi-\xi^{\prime}$ and, by hypothesis, (14) holds, we have

$$
\bigoplus_{j \leq 2 i+1} \mathfrak{g}_{j}^{\xi} \cap \mathfrak{k}_{\sigma}^{\mathbb{C}}=\left(\bigoplus_{j \leq 2 i} \mathfrak{g}_{j}^{\xi} \cap \mathfrak{k}_{\sigma}^{\mathbb{C}}\right) \oplus\left(\mathfrak{g}_{2 i+1}^{\xi} \cap \mathfrak{k}_{\sigma}^{\mathbb{C}}\right) \subset\left(\bigoplus_{j \leq 2 i} \mathfrak{g}_{j}^{\xi-\xi^{\prime}} \cap \mathfrak{k}_{\sigma}^{\mathbb{C}}\right) \oplus \bigoplus_{0 \leq j<2 i+1} \mathfrak{g}_{j}^{\xi-\xi^{\prime}} .
$$

Hence the component of $X_{2 i}^{\prime}$ over $\mathfrak{g}_{2 i+1}^{\xi-\xi^{\prime}}$ vanishes for all $i \geq 0$. Similarly, for $r=2 i-1, X_{2 i-1}^{\prime}$ takes values in $\bigoplus_{j \leq 2 i} \mathfrak{g}_{j}^{\xi} \cap \mathfrak{m}_{\sigma}^{\mathbb{C}}$, and we can check that the component of $X_{2 i-1}^{\prime}$ over $\mathfrak{g}_{2 i}^{\xi-\xi^{\prime}}$ vanishes for all $i>0$.

Hence $\gamma^{-1}:=\mathcal{U}_{\xi, \xi-\xi^{\prime}}(\Phi)=\Psi \cdot \gamma_{\xi-\xi^{\prime}}$ is a constant loop.

Definition 2. We say that $\zeta \in \mathfrak{I}^{\prime}\left(G^{\sigma_{\varrho}}\right) \cap \mathfrak{C}_{I}^{\varrho}$ is a $\varrho$-semi-canonical element if $\zeta$ is of the form $\zeta=\sum_{i \in I} n_{i} \zeta_{i}$ with $1 \leq n_{i} \leq 2 m_{i}$, where $m_{i}$ is the least positive integer which makes $m_{i} \zeta_{i} \in \mathfrak{I}^{\prime}\left(G^{\sigma_{\varrho}}\right)$.

Corollary 1. Take $\xi \in \mathfrak{I}^{\prime}\left(G^{\sigma_{\varrho}}\right) \cap \mathfrak{C}_{I}^{\varrho}$, with $I \subset\{1, \ldots, k\}$. Let $\Phi: S^{2} \backslash D \rightarrow U_{\xi}^{\sigma_{\varrho}}(G)$ be a $T_{\sigma_{\varrho}}$-invariant extended solution, and let $\varphi: S^{2} \rightarrow P_{\xi}^{\sigma_{\varrho}}$ be the corresponding harmonic map. Then there exist $h \in G$, a constant loop $\gamma$, and a $\varrho$-semi-canonical $\zeta$ such that $\tilde{\Phi}:=h \gamma \Phi h^{-1}$ defined on $S^{2} \backslash D$ takes values in $U_{\zeta}^{\sigma_{\varrho}}(G)$. The harmonic map $\tilde{\Phi}_{-1}$ takes values in $P_{\zeta}^{\sigma_{\varrho}}=P_{\xi}^{\sigma_{\varrho}}$ and coincides with $\varphi$ up to isometry. 
N. CORREIA AND R. PACHECO

Proof. Write $\xi=\sum_{i \in I} r_{i} \zeta_{i}$, with $r_{i}>0$. For each $i \in I$, let $n_{i}$ be the unique integer number in $\left\{1, \ldots, 2 m_{i}\right\}$ such that $n_{i}=r_{i} \bmod 2 m_{i}$. Set $\zeta=\sum_{i \in I} n_{i} \zeta_{i}$. It is clear that $\xi \preceq \zeta$ and $\zeta \in \mathfrak{I}^{\prime}\left(G^{\sigma_{\varrho}}\right) \cap \mathfrak{C}_{I}^{\varrho}$. Observe also that conditions (14) hold automatically for any $\xi^{\prime} \in \mathfrak{I}^{\prime}\left(G^{\sigma_{\varrho}}\right) \cap \mathfrak{C}_{I}^{\circ}$ satisfying $\xi \preceq \xi^{\prime}$. In particular they hold for $\xi^{\prime}=\zeta$. Finally, since $\xi-\zeta=2 \sum_{i \in I} m_{i} k_{i} \zeta_{i}$ for some nonnegative integer numbers $k_{i}$, then $\exp \pi(\xi-\zeta)=e$, and the result follows from Propositions 15 and 16.

4.2.3. Classification of harmonic two-spheres into outer symmetric spaces. To sum up, in order to classify all harmonic two-spheres into outer symmetric spaces we proceed as follows:

(1) Start with a fundamental outer involution $\sigma_{\varrho}$ and let $N$ be an outer symmetric $G$-space corresponding to an involution of the form $\sigma_{\varrho}$ or $\sigma_{\varrho, i}$ of $G$, according to (10), where the element $\zeta_{i}$ is in the conditions of Theorem 17. We assume that $\exp 2 \pi \zeta_{i}=e$, that is $\zeta_{i} \in \mathfrak{I}^{\prime}\left(G^{\sigma_{\varrho}}\right)$. Let $\varphi: S^{2} \rightarrow N$ be an harmonic map and identify $N$ with $P_{\zeta_{i}}^{\sigma_{Q}}=\exp \left(\pi \zeta_{i}\right) P_{e}^{\sigma_{Q}, i}$ via the totally geodesic embedding (13). If $N$ is the fundamental outer space with involution $\sigma_{\varrho}$ we simply identify $N$ with $P_{e}^{\sigma_{\varrho}}$ via $\iota_{\sigma_{e}}$.

(2) By Theorem 12. $\varphi: S^{2} \rightarrow N \cong P_{\zeta_{i}}^{\sigma_{\varrho}}$ admits a $T_{\sigma_{\varrho}}$-invariant extended solution $\Phi: S^{2} \rightarrow \Omega^{\sigma_{\varrho}} G$ which takes values, off some discrete subset $D$, in some unstable manifold $U_{\zeta^{\prime}}^{\sigma_{o}}(G)$, with $\zeta^{\prime} \in \mathfrak{I}^{\prime}\left(G^{\sigma_{\varrho}}\right)$; moreover, $P_{\zeta^{\prime}}^{\sigma_{e}}=P_{\zeta_{i}}^{\sigma_{e}}$.

(3) By Corollary 1 we can assume that $\zeta^{\prime}$ is a $\varrho$-semi-canonical element in $\mathfrak{I}^{\prime}\left(G^{\sigma_{\varrho}}\right) \cap \mathfrak{C}_{I}^{\varrho}$. If $\zeta$ is a $\varrho$-canonical element such that $\zeta^{\prime} \preceq \zeta$ and $\mathcal{U}_{\zeta^{\prime}, \zeta^{\prime}-\zeta}(\Phi)$ is constant, then, taking into account Proposition 15, there exists a $T_{\tau}$-invariant extended solution $\tilde{\Phi}: S^{2} \backslash D \rightarrow U_{\zeta}^{\tau}(G)$, where

$$
\tau=\operatorname{Ad}\left(\exp \pi\left(\zeta^{\prime}-\zeta\right)\right) \circ \sigma_{\varrho}
$$

such that the harmonic map $\varphi$ is given, up to isometry, by $\tilde{\Phi}_{-1}: S^{2} \rightarrow P_{\zeta}^{\tau}$. Here we identify $N$ with $P_{\zeta}^{\tau}=\exp \left(\pi\left(\zeta^{\prime}-\zeta\right)\right) P_{\zeta_{i}}^{\sigma_{Q}}$ via the composition of (13) with the left multiplication by $\exp \left(\pi\left(\zeta^{\prime}-\zeta\right)\right)$.

(4) By Proposition 16, there always exists a $\varrho$-canonical element $\zeta$ in such conditions.

Hence, we classify harmonic spheres into outer symmetric $G$-spaces in terms of pairs $(\zeta, \tau)$, where $\zeta$ is a $\varrho$-canonical element and $\tau$ is an outer involution of the form (16) for some $\varrho$-semi-canonical element $\zeta^{\prime}$ with $\zeta^{\prime} \preceq \zeta$.

4.2.4. Weierstrass Representation for $T_{\sigma}$-invariant Extended Solutions. From (15) and Proposition 4 , we obtain the following.

Theorem 17. Let $\Phi: M \rightarrow \Omega_{\mathrm{alg}}^{\sigma} G$ be an extended solution. There exists a discrete set $D^{\prime} \supseteq D$ of $M$ such that $\left.\Phi\right|_{M \backslash D^{\prime}}=\exp C \cdot \gamma_{\xi}$ for some holomorphic vector-valued function $C: M \backslash D^{\prime} \rightarrow\left(\mathfrak{u}_{\xi}^{0}\right)_{\sigma}$, where $\left(\mathfrak{u}_{\xi}^{0}\right)_{\sigma}$ is the finite dimensional nilpotent subalgebra of $\Lambda_{\text {alg }}^{+} \mathfrak{g}^{\mathbb{C}}$ defined by

$$
\left(\mathfrak{u}_{\xi}^{0}\right)_{\sigma}=\bigoplus_{0 \leq 2 i<r(\xi)} \lambda^{2 i}\left(\mathfrak{p}_{2 i}^{\xi}\right)^{\perp} \cap \mathfrak{k}_{\sigma}^{\mathbb{C}} \oplus \bigoplus_{0 \leq 2 i+1<r(\xi)} \lambda^{2 i+1}\left(\mathfrak{p}_{2 i+1}^{\xi}\right)^{\perp} \cap \mathfrak{m}_{\sigma}^{\mathbb{C}},
$$

with $\left(\mathfrak{p}_{i}^{\xi}\right)^{\perp}=\bigoplus_{i<j \leq r(\xi)} \mathfrak{g}_{j}^{\xi}$. Moreover, $C$ can be extended meromorphically to $M$.

\section{EXAMPLES}

Next we will describe explicit examples of harmonic spheres into classical outer symmetric spaces.

5.1. Outer symmetric $S O(2 n)$-spaces. For details on the structure of $\mathfrak{s o}(2 n)$ see [10]. Consider on $\mathbb{R}^{2 n}$ the standard inner product $\langle\cdot, \cdot\rangle$ and fix a complex basis $\mathbf{u}=\left\{u_{1}, \ldots, u_{n}, \bar{u}_{1}, \ldots, \bar{u}_{n}\right\}$ of $\mathbb{C}^{2 n}=\left(\mathbb{R}^{2 n}\right)^{\mathbb{C}}$ satisfying

$$
\left\langle u_{i}, u_{j}\right\rangle=0, \quad\left\langle u_{i}, \bar{u}_{j}\right\rangle=\delta_{i j}, \quad \text { for all } 1 \leq i, j \leq n .
$$

Throughout this section we will denote by $V_{l}$ the $l$-dimensional isotropic subspace spanned by $\bar{u}_{1}, \ldots, \bar{u}_{l}$.

Set $E_{i}=E_{i, i}-E_{n+i, n+i}$, where $E_{j, j}$ is a square matrix, with respect to the basis $\mathbf{u}$, whose $(j, j)$-entry is i and all other entries are 0 . The complexification $\mathfrak{t}^{\mathbb{C}}$ of the algebra $\mathfrak{t}$ of diagonal matrices $\sum a_{i} E_{i}$, with $a_{i} \in \mathbb{R}$ 
and $\sum a_{i}=0$, is a Cartan subalgebra of $\mathfrak{s o}(2 n)^{\mathbb{C}}$. Let $\left\{L_{1}, \ldots, L_{n}\right\}$ be the dual basis in it* of $\left\{E_{1}, \ldots, E_{n}\right\}$, that is $L_{i}\left(E_{j}\right)=\mathrm{i} \delta_{i j}$. The roots of $\mathfrak{s o}(2 n)$ are the vectors $\pm L_{i} \pm L_{j}$ and $\pm L_{i} \mp L_{j}$, with $i \neq j$ and $1 \leq i, j \leq n$.

Consider the endomorphisms

$$
X_{i, j}=E_{i, j}-E_{n+j, n+i}, Y_{i, j}=E_{i, n+j}-E_{j, n+i}, Z_{i, j}=E_{n+i, j}-E_{n+j, i},
$$

where $E_{i, j}$, with $i \neq j$, is a square matrix whose $(i, j)$-entry is 1 and all other entries are 0 . The root spaces of $L_{i}-L_{j}, L_{i}+L_{j}$ and $-L_{i}-L_{j}$, respectively, are generated by the endomorphisms $X_{i, j}, Y_{i, j}$ and $Z_{i, j}$, respectively.

Fix the positive root system $\Delta^{+}=\left\{L_{i} \pm L_{j}\right\}_{i<j}$. The positive simple roots are $\alpha_{i}=L_{i}-L_{i+1}$, for $1 \leq i \leq n-1$, and $\alpha_{n}=L_{n-1}+L_{n}$. The vectors of the dual basis $\left\{H_{1}, \ldots, H_{n}\right\} \subset \mathfrak{t}$ are given by $H_{i}=E_{1}+E_{2}+\ldots+E_{i}$, for $1 \leq i \leq n-2$,

$$
H_{n-1}=\frac{1}{2}\left(E_{1}+E_{2}+\ldots+E_{n-1}-E_{n}\right), \text { and } H_{n}=\frac{1}{2}\left(E_{1}+E_{2}+\ldots+E_{n-1}+E_{n}\right) .
$$

Consider the non-trivial involution $\varrho$ of the corresponding Dynkin diagram,

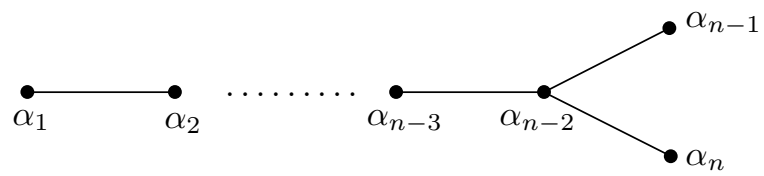

This involution fixes $\alpha_{i}$ if $i \leq n-2$ and $\varrho\left(\alpha_{n-1}\right)=\alpha_{n}$. The corresponding semi-fundamental basis $\pi_{\mathfrak{k}_{\varrho}}\left(\Delta_{0}\right)=$ $\left\{\beta_{1}, \ldots, \beta_{n-1}\right\}$ is given by

$$
\beta_{i}=\alpha_{i}=L_{i}-L_{i+1} \text {, if } i \leq n-2, \text { and } \beta_{n-1}=\frac{1}{2}\left(\alpha_{n-1}+\alpha_{n}\right)=L_{n-1},
$$

whereas the dual basis $\left\{\zeta_{1}, \ldots, \zeta_{n-1}\right\}$ is given by $\zeta_{i}=E_{1}+\ldots+E_{i}$, with $i=1, \ldots, n-1$. Since each $\zeta_{i}$ belongs to the integer lattice $\mathfrak{I}\left(S O(2 n)^{\sigma_{\varrho}}\right)$, we have:

Proposition 18. The $\varrho$-semi-canonical elements of $S O(2 n)$ are precisely the elements $\zeta=\sum_{i=1}^{n-1} m_{i} \zeta_{i}$ such that $m_{i} \in\{0,1,2\}$ for $1 \leq i \leq n-1$.

The fundamental outer symmetric $S O(2 n)$-space is the real projective space $\mathbb{R} P^{2 n-1}$, and the associated outer symmetric $S O(2 n)$-spaces are the real Grassmannians $G_{p}\left(\mathbb{R}^{2 n}\right)$ with $p>1$ odd.

5.1.1. Harmonic maps into real projective spaces $\mathbb{R} P^{2 n-1}$. Consider as base point the one dimensional real vector space $V_{0}$ spanned by $e_{n}=\left(u_{n}+\bar{u}_{n}\right) / \sqrt{2}$ in $\mathbb{R}^{2 n}$, which establishes an identification of $\mathbb{R} P^{2 n-1}$ with $S O(2 n) / S(O(1) O(2 n-1))$. Denote by $\pi_{V_{0}}$ and $\pi_{V_{0}}^{\perp}$ the orthogonal projections onto $V_{0}$ and $V_{0}^{\perp}$, respectively. The fundamental involution is given by $\sigma_{\varrho}=\operatorname{Ad}\left(s_{0}\right)$, where $s_{0}=\pi_{V_{0}}-\pi_{V_{0}}^{\perp}$. Following the classification procedure established in Section 4.2.3 we start by identifying $\mathbb{R} P^{2 n-1}$ with $P_{e}^{\sigma_{e}}$.

Theorem 19. Each harmonic map $\varphi: S^{2} \rightarrow \mathbb{R} P^{2 n-1}$ belongs to one of the following classes: $\left(\zeta_{l}, \sigma_{\varrho, l}\right)$, with $1 \leq l \leq n-1$.

Proof. Let $\zeta$ be a $\varrho$-semi-canonical element and write

$$
\zeta=\sum_{i \in I_{1}} \zeta_{i}+\sum_{i \in I_{2}} 2 \zeta_{i}
$$

for some disjoint subsets $I_{1}$ and $I_{2}$ of $\{1, \ldots, n-1\}$. By Proposition $9 P_{\zeta}^{\sigma_{\varrho}} \cong \mathbb{R} P^{2 n-1}$ if and only if either $I_{1}=\emptyset$ or $I_{1}=\{n-1\}$. Suppose that $I_{1}=\{n-1\}$. In this case, $\exp \pi \zeta=\exp \pi \zeta_{n-1} \in P_{\zeta_{n-1}}^{\sigma_{\varrho}}$. We claim that $P_{\zeta_{n-1}}^{\sigma_{\varrho}}$ is not the connected component of $P^{\sigma_{\varrho}}$ containing the identity $e$. Write $\exp \pi \zeta_{n-1}=\pi_{V}-\pi_{V}^{\perp}$, where $V$ is the two-dimensional real space spanned by $e_{n}$ and $e_{2 n}$. For each $g \in P_{e}^{\sigma_{\varrho}}$, since the $G$-action $\cdot_{\sigma_{\varrho}}$ defined by (3) is transitive, we have $g=h \cdot_{\sigma_{e}} e=h s_{0} h^{-1} s_{0}$ for some $h \in G$, which means that $g s_{0}=h s_{0} h^{-1}$. In particular, the +1-eigenspaces of $g s_{0}$ must be 1-dimensional. However, a simple computation shows that the +1-eigenspace of $\exp \left(\pi \zeta_{n-1}\right) s_{0}$ is 3 -dimensional, which establishes our claim. 
Then, any harmonic map $\varphi: S^{2} \rightarrow \mathbb{R} P^{2 n-1} \cong P_{e}^{\sigma_{\varrho}}$ admits a $T_{\sigma_{e}}$-invariant extended solution $\Phi: S^{2} \backslash D \rightarrow$ $U_{\zeta}^{\sigma_{\varrho}}(S O(2 n))$ with $\zeta$ a $\varrho$-semi-canonical element of the form $\zeta=\sum_{i \in I_{2}} 2 \zeta_{i}$. Set $l=\max I_{2}$. Next we check that $\zeta$ and $\zeta_{l}$ satisfy the conditions of Proposition [16, with $\xi=\zeta$ and $\xi^{\prime}=\zeta_{l}$. It is clear that $\zeta \preceq \zeta_{l}$. Now, according to (11) and (12), we can take $\Delta_{\varrho}^{\prime}=\left\{L_{i}-L_{n}, L_{n}-L_{i}\right\}$. Hence, for $i>0$,

$$
\mathfrak{g}_{2 i}^{\zeta} \cap \mathfrak{m}_{\varrho}^{\mathbb{C}}=\bigoplus_{\alpha \in \Delta_{\varrho}^{\prime} \cap \Delta_{\zeta}^{2 i}}\left(\mathfrak{g}_{\alpha} \oplus \mathfrak{g}_{\varrho(\alpha)}\right) \cap \mathfrak{m}_{\varrho}^{\mathbb{C}}
$$

where $\Delta_{\zeta}^{2 i}=\{\alpha \in \Delta \mid \alpha(\zeta)=2 i \mathrm{i}\}$. Since

$$
\left(L_{j}-L_{n}\right)(\zeta)=\left(\alpha_{j}+\alpha_{j+1}+\ldots+\alpha_{n-1}\right)(\zeta)=2\left|I_{2} \cap\{j, \ldots, n-1\}\right| \mathrm{i},
$$

we have

$$
\Delta_{\varrho}^{\prime} \cap \Delta_{\zeta}^{2 i}=\left\{L_{j}-L_{n} \mid 1 \leq j \leq l, \text { and }\left|I_{2} \cap\{j, \ldots, l\}\right|=i\right\} .
$$

Then, given a root $\alpha=L_{j}-L_{n} \in \Delta_{\varrho}^{\prime} \cap \Delta_{\zeta}^{2 i}$ (in particular, $\left.j \leq l\right)$ we have $\alpha\left(\zeta-\zeta_{l}\right)=(2 i-1)$ i, which means that $\mathfrak{g}_{\alpha} \subset \mathfrak{g}_{2 i-1}^{\zeta-\zeta_{l}}$. Consequently,

$$
\mathfrak{g}_{2 i}^{\zeta} \cap \mathfrak{m}_{\varrho}^{\mathbb{C}} \subset \bigoplus_{0 \leq j<2 i} \mathfrak{g}_{j}^{\zeta-\zeta_{l}}
$$

Since $\mathfrak{g}_{2 i-1}^{\zeta}=\{0\}$ for all $i$, we conclude that (14) holds, and the statement follows from Propositions [15] and 16.

It is known [3] that there are no full harmonic maps $\varphi: S^{2} \rightarrow \mathbb{R} P^{2 n-1}$. The class of harmonic maps associated to $\left(\zeta_{l}, \sigma_{\varrho, l}\right)$ consists precisely of those $\varphi$ with $\varphi\left(S^{2}\right)$ contained, up to isometry, in some $\mathbb{R} P^{2 l}$, as shown in the next theorem.

Theorem 20. Given $1 \leq l \leq n-1$, any harmonic map $\varphi: S^{2} \rightarrow \mathbb{R} P^{2 n-1}$ in the class $\left(\zeta_{l}, \sigma_{\varrho, l}\right)$ is given by

$$
\varphi=R \cap(A \oplus \bar{A})^{\perp},
$$

where $R$ is a constant $2 l+1$-dimensional subspace of $\mathbb{R}^{2 n}$ and $A$ is a holomorphic isotropic subbundle of $S^{2} \times R$ of rank $l$ satisfying $\partial A \subseteq \bar{A}^{\perp}$. The corresponding extended solutions have uniton number 2 with respect to the standard representation of $S O(2 n)$.

Proof. Let $\varphi: S^{2} \rightarrow \mathbb{R} P^{2 n-1}$ be a harmonic map in the class $\left(\zeta_{l}, \sigma_{\varrho, l}\right)$. This means that $\varphi$ admits an extended solution $\Phi: S^{2} \backslash D \rightarrow U_{\zeta_{l}}^{\sigma_{\varrho, l}}(S O(2 n))$. Up to isometry, $\varphi$ is given by $\Phi_{-1}$, which takes values in $P_{\zeta_{l}, l}^{\sigma_{Q}}=$ $\exp \left(\pi \zeta_{l}\right) P_{e}^{\sigma_{Q}}$. This connected component is identified with $\mathbb{R} P^{2 n-1}$ via

$$
g \cdot V_{0} \mapsto \exp \left(\pi \zeta_{l}\right) g \sigma_{\varrho}\left(g^{-1}\right)
$$

Write $\gamma_{\zeta_{l}}(\lambda)=\lambda^{-1} \pi_{V_{l}}+\pi_{V_{l} \oplus \bar{V}_{l}}^{\perp}+\lambda \pi_{\bar{V}_{l}}$, where $V_{l}$ is the $l$-dimensional isotropic subspace spanned by $\bar{u}_{1}, \ldots, \bar{u}_{l}$.

We have $r\left(\zeta_{l}\right)=2$ if $l>1$ and $r\left(\zeta_{1}\right)=1$. Consequently, by Theorem 17

$$
\left(\mathfrak{u}_{\zeta_{l}}^{0}\right)_{\sigma_{\varrho, l}}=\left(\mathfrak{p}_{0}^{\zeta l}\right)^{\perp} \cap \mathfrak{k}_{\sigma_{e, l}}^{\mathbb{C}} \oplus \lambda\left(\mathfrak{p}_{1}^{\zeta l}\right)^{\perp} \cap \mathfrak{m}_{\sigma_{e, l}}^{\mathbb{C}} .
$$

Here $\left(\mathfrak{p}_{1}^{\zeta_{l}}\right)^{\perp}=\mathfrak{g}_{2}^{\zeta_{l}}$, which is the null space for $l=1$. For $l>1$, since $\zeta_{l}=E_{1}+\ldots+E_{l}$, we have $\mathfrak{g}_{2}^{\zeta_{l}}=$ $\left\{L_{i}+L_{j} \mid 1 \leq i<j \leq l\right\} \subset \Delta\left(\mathfrak{k}_{\varrho}\right)$ and, from (6),

$$
\mathfrak{m}_{\sigma_{\varrho}, l}^{\mathbb{C}}=\bigoplus \mathfrak{g}_{2 i+1}^{\zeta_{l}} \cap \mathfrak{k}_{\varrho}^{\mathbb{C}} \oplus \bigoplus \mathfrak{g}_{2 i}^{\zeta_{l}} \cap \mathfrak{m}_{\varrho}^{\mathbb{C}} .
$$

Hence $\left(\mathfrak{p}_{1}^{\zeta_{l}}\right)^{\perp} \cap \mathfrak{m}_{\sigma_{e, l}}^{\mathbb{C}}=\mathfrak{g}_{2}^{\zeta_{l}} \cap \mathfrak{m}_{\varrho}^{\mathbb{C}}=\{0\}$. Then, for any $l \geq 1$, we can write $\Phi=\exp C \cdot \gamma_{\zeta_{l}}$ for some holomorphic function

$$
C: S^{2} \backslash D \rightarrow\left(\mathfrak{p}_{0}^{\zeta_{l}}\right)^{\perp} \cap \mathfrak{k}_{\sigma_{e, l}}^{\mathbb{C}}=\left(\mathfrak{g}_{1}^{\zeta_{l}} \oplus \mathfrak{g}_{2}^{\zeta_{l}}\right) \cap \mathfrak{k}_{\sigma_{e, l}}^{\mathbb{C}},
$$

which means that $\Phi$ is a $S^{1}$-invariant extended solution with uniton number 2 :

$$
\Phi_{\lambda}=\lambda^{-1} \pi_{W}+\pi_{W \oplus \bar{W}}^{\perp}+\lambda \pi_{\bar{W}},
$$


where $W$ is a holomorphic isotropic subbundle of $S^{2} \times \mathbb{R}^{2 n}$ of rank $l$ satisfying the superhorizontality condition $\partial W \subseteq \bar{W}^{\perp}$.

Set $\tilde{V}_{l}=V_{l} \oplus \bar{V}_{l}$ and $\tilde{W}=W \oplus \bar{W}$. The $T_{\sigma_{e, l}}$ invariance of $\Phi$ implies that

$$
\left[\pi_{W}, \pi_{V_{0} \oplus \tilde{V}_{l}}\right]=0 .
$$

Now, write $\varphi=g \cdot V_{0}$ and consider the identification (20). We must have

$$
\Phi_{-1}=\exp \left(\pi \zeta_{l}\right) g \sigma_{\varrho}\left(g^{-1}\right)=\exp \left(\pi \zeta_{l}\right)\left(\pi_{\varphi}-\pi_{\varphi}^{\perp}\right) s_{0} .
$$

From (21) and (23) we obtain

$$
\pi_{\varphi}-\pi_{\varphi}^{\perp}=\operatorname{Ad}\left(s_{0}\right)\left(\pi_{V_{0} \oplus \tilde{V}_{l}} \pi_{\tilde{W}}^{\perp}+\pi_{V_{0} \oplus \tilde{V}_{l}}^{\perp} \pi_{\tilde{W}}-\pi_{V_{0} \oplus \tilde{V}_{l}} \pi_{\tilde{W}}-\pi_{V_{0} \oplus \tilde{V}_{l}}^{\perp} \pi_{\tilde{W}}^{\perp}\right) .
$$

In view of (22), we see that $\pi_{V_{0} \oplus \tilde{V}_{l}} \pi_{\tilde{W}}^{\perp}+\pi_{V_{0} \oplus \tilde{V}_{l}}^{\perp} \pi_{\tilde{W}}$ is an orthogonal projection, and (24) implies that this must be an orthogonal projection onto a 1-dimensional real subspace. Then, one of its two terms vanishes, that is either $\tilde{W} \subset V_{0} \oplus \tilde{V}_{l}$ or $\tilde{W}^{\perp} \subset\left(V_{0} \oplus \tilde{V}_{l}\right)^{\perp}$. For dimensional reasons, we see that the second case can not occur. Hence, we have

$$
\pi_{\varphi}=\operatorname{Ad}\left(s_{0}\right)\left(\pi_{V_{0} \oplus \tilde{V}_{l}} \pi_{\tilde{W}}^{\perp}\right)=\pi_{V_{0} \oplus \tilde{V}_{l}} \operatorname{Ad}\left(s_{0}\right)\left(\pi_{\tilde{W}}^{\perp}\right),
$$

that is (19) holds with $R=V_{0} \oplus V_{l} \oplus \bar{V}_{l}$ and $A=s_{0}(W)$.

Remark 4. If $\varphi$ is full in $R$, then the isotropic subbundle $A$ is the l-osculating space of some full totally isotropic holomorphic map $f$ from $S^{2}$ into the complex projective space of $R$, the so called directrix curve of $\varphi$. That is, in a local system of coordinates $(U, z)$, we have $A(z)=\operatorname{Span}\left\{g, g^{\prime}, \ldots, g^{(l-1)}\right\}$, where $g$ is a lift of $f$ over $U$ and $g^{(r)}$ the $r$-th derivative of $g$ with respect to $z$. Hence, formula (19) agrees with the classification given in Corollary 6.11 of $[9]$.

Example 1. Let us consider the case $n=2$. We have only one class of harmonic maps: $\left(\zeta_{1}, \sigma_{\varrho, 1}\right)$. From Theorem 20, any such harmonic map $\varphi: S^{2} \rightarrow \mathbb{R} P^{3}$ is given by $\varphi=R \cap(A \oplus \bar{A})^{\perp}$, where $R$ is a constant 3-dimensional subspace of $\mathbb{R}^{4}$ and $A$ a holomorphic isotropic subbundle of $S^{2} \times R$ of rank 1 such that $\partial A \subseteq \bar{A}^{\perp}$. Taking into account Theorem 17, any such holomorphic subbundles $A$ can be obtained from a meromorphic function $a$ on $S^{2}$ as follows.

We have $\zeta_{1}=E_{1}$ and the corresponding extended solutions have uniton number $r\left(\zeta_{1}\right)=1$ (with respect to the standard representation). Any extended solution $\Phi: S^{2} \backslash D \rightarrow U_{\zeta_{1}}^{\sigma_{e, 1}}(S O(4))$ is given by $\Phi=\exp C \cdot \gamma_{\zeta_{1}}$, with $\gamma_{\zeta_{1}}(\lambda)=\lambda^{-1} \pi_{V_{1}}+\pi_{V_{1} \oplus \bar{V}_{1}}^{\perp}+\lambda \pi_{\bar{V}_{1}}$, for some holomorphic vector-valued function $C: S^{2} \backslash D \rightarrow\left(\mathfrak{u}_{\zeta_{1}}^{0}\right)_{\sigma_{Q, 1}}$, where

$$
\left(\mathfrak{u}_{\zeta_{1}}^{0}\right)_{\sigma_{Q, 1}}=\left(\mathfrak{p}_{0}^{\zeta_{1}}\right)^{\perp} \cap \mathfrak{k}_{\sigma_{Q, 1}}^{\mathbb{C}}=\mathfrak{g}_{1}^{\zeta_{1}} \cap \mathfrak{k}_{\sigma_{Q, 1}}^{\mathbb{C}}=\left(\mathfrak{g}_{L_{1}-L_{2}} \oplus \mathfrak{g}_{L_{1}+L_{2}}\right) \cap \mathfrak{k}_{\sigma_{Q, 1}}^{\mathbb{C}} .
$$

Considering the root vectors $X_{i, j}, Y_{i, j}, Z_{i, j}$ as defined in (17), we have $Y_{1,2}=\sigma_{\varrho, 1}\left(X_{1,2}\right)$. Hence $C=$ $a(z)\left(X_{1,2}+Y_{1,2}\right)$ where $a(z)$ is a meromorphic function on $S^{2}$. In this case, from (2) , it follows that $(\exp C)^{-1}(\exp C)_{z}=$ $C_{z}$, and it is clear that the extended solution condition for $\Phi$ holds independently of the choice of the meromorphic function $a(z)$. Then, with respect to the complex basis $\mathbf{u}=\left\{u_{1}, u_{2}, \bar{u}_{1}, \bar{u}_{2}\right\}$,

$$
\exp C \cdot \gamma_{\zeta_{1}}=\left[\begin{array}{cccc}
1 & a & -a^{2} & a \\
0 & 1 & -a & 0 \\
0 & 0 & 1 & 0 \\
0 & 0 & -a & 1
\end{array}\right] \cdot \gamma_{\zeta_{1}}
$$

and the subbundle $A$ of $R=\operatorname{Span}\left\{u_{1}, \bar{u}_{1}, u_{2}+\bar{u}_{2}\right\}$ is given by $A=\exp C \cdot V_{1}=\operatorname{span}\left\{\left(a^{2}, a,-1, a\right)\right\}$, which satisfies $\partial A \subseteq \bar{A}^{\perp}$. 
Example 2. Any harmonic two-sphere into $\mathbb{R} P^{5}$ in the class $\left(\zeta_{1}, \sigma_{\varrho, 1}\right)$ takes values in some $\mathbb{R} P^{3}$ inside $\mathbb{R} P^{5}$ and so it is essentially of the form (25). Next we consider the Weierstrass representation of harmonic spheres into $\mathbb{R} P^{5}$ in the class $\left(\zeta_{2}, \sigma_{\varrho, 2}\right)$, which are given by $\varphi=R \cap(A \oplus \bar{A})^{\perp}$, where $R$ is a constant 5-dimensional subspace of $\mathbb{R}^{6}$ and $A$ a holomorphic isotropic subbundle of $S^{2} \times R$ of rank 2 such that $\partial A \subseteq \bar{A}^{\perp}$. We have $\zeta_{2}=E_{1}+E_{2}$, then $r\left(\zeta_{2}\right)=2$. Any extended solution $\Phi: S^{2} \backslash D \rightarrow U_{\zeta_{2}, 2}^{\sigma_{\varrho}}(S O(6))$ is given by $\Phi=\exp C \cdot \gamma_{\zeta_{2}}$, with $\gamma_{\zeta_{2}}(\lambda)=\lambda^{-1} \pi_{V_{2}}+\pi_{V_{2} \oplus \bar{V}_{2}}^{\perp}+\lambda \pi_{\bar{V}_{2}}$, for some holomorphic vector-valued function $C: S^{2} \backslash D \rightarrow\left(\mathfrak{u}_{\zeta_{2}}^{0}\right)_{\sigma_{\varrho}, 2}$, where

$$
\left(\mathfrak{u}_{\zeta_{2}}^{0}\right)_{\sigma_{\varrho, 2}}=\left(\left(\mathfrak{g}_{L_{1}-L_{3}} \oplus \mathfrak{g}_{L_{1}+L_{3}}\right) \cap \mathfrak{k}_{\sigma_{\varrho, 2}}^{\mathbb{C}}\right) \oplus\left(\left(\mathfrak{g}_{L_{2}-L_{3}} \oplus \mathfrak{g}_{L_{2}+L_{3}}\right) \cap \mathfrak{k}_{\sigma_{\varrho, 2}}^{\mathbb{C}}\right) \oplus \mathfrak{g}_{L_{1}+L_{2}} .
$$

We have $Y_{1,3}=\sigma_{\varrho, 2}\left(X_{1,3}\right)$ and $Y_{2,3}=\sigma_{\varrho, 2}\left(X_{2,3}\right)$. Hence we can write

$$
C=a(z)\left(X_{1,3}+Y_{1,3}\right)+b(z)\left(X_{2,3}+Y_{2,3}\right)+c(z) Y_{1,2}
$$

where $a(z), b(z)$ and $c(z)$ are meromorphic functions on $S^{2}$.

Now, $\Phi=\exp C \cdot \gamma_{\zeta_{2}}$ is an extended solution if and only if, in the expression $C_{z}-\frac{1}{2 !}(\operatorname{ad} C) C_{z}$, which does not depend on $\lambda$, the component on $\mathfrak{g}_{2}^{\zeta_{2}}=\mathfrak{g}_{L_{1}+L_{2}}$ vanishes. Since $Y_{1,2}=\left[Y_{2,3}, X_{1,3}\right]=\left[X_{2,3}, Y_{1,3}\right]$ and $\left[X_{1,3}, X_{2,3}\right]=\left[Y_{1,3}, Y_{2,3}\right]=0$, this holds if and only if $c^{\prime}=b a^{\prime}-a b^{\prime}$, where prime denotes $z$-derivative. Since $A=\exp C \cdot V_{2}$, we can compute $\exp C$ in order to conclude that the holomorphic subbundle $A$ of $R=\operatorname{Span}\left\{u_{1}, u_{2}, \bar{u}_{1}, \bar{u}_{2}, u_{3}+\bar{u}_{3}\right\}$ is given by

$$
A=\operatorname{Span}\left\{\left(a^{2}, a b+c, a,-1,0, a\right),\left(a b-c, b^{2}, b, 0,-1, b\right)\right\} .
$$

5.1.2. Harmonic maps into Real Grassmanians. Let $\zeta^{\prime}$ be a $\varrho$-semi-canonical element of $S O(2 n)$ given by (18), for some disjoint subsets $I_{1}$ and $I_{2}$ of $\{1, \ldots, n-1\}$. By Proposition 9 , we know that $P_{\zeta^{\prime}}^{\sigma} \cong \mathbb{R} P^{2 n-1}$ if and only if either $I_{1}=\emptyset$ or $I_{1}=\{n-1\}$. More generally we have:

Proposition 21. If $I_{1}=\left\{i_{1}>i_{2}>\ldots>i_{r}\right\}$ and $d=\sum_{j=1}^{r}(-1)^{j+1} i_{j}$, then $P_{\zeta^{\prime}}^{\sigma_{\varrho}} \cong G_{2 d+1}\left(\mathbb{R}^{2 n}\right)$.

Proof. For $\zeta^{\prime}$ of the form (18), set $\zeta_{I_{1}}^{\prime}=\sum_{i \in I_{1}} \zeta_{i}$. Clearly, $\exp \pi \zeta^{\prime}=\exp \pi \zeta_{I_{1}}^{\prime}$, and, by Proposition 6 , $P_{\zeta^{\prime}}^{\sigma_{\varrho}}$ is a symmetric space with involution

$$
\tau=\operatorname{Ad}\left(\exp \pi \zeta_{I_{1}}^{\prime}\right) \circ \sigma_{\varrho}=\operatorname{Ad}\left(s_{0} \exp \pi \zeta_{I_{1}}^{\prime}\right)
$$

We have

$$
\zeta_{I_{1}}^{\prime}=r\left(E_{1}+\ldots+E_{i_{r}}\right)+(r-1)\left(E_{i_{r}+1}+\ldots+E_{i_{r-1}}\right)+\ldots+\left(E_{i_{2}+1}+\ldots+E_{i_{1}}\right),
$$

and consequently, with the convention $V_{i_{0}}=V_{n}$ and $V_{i_{r+1}}=\{0\}$,

$$
\exp \pi \zeta_{I_{1}}^{\prime}=\sum_{j=0}^{r}(-1)^{j} \pi_{i_{j}-i_{j+1}}+\sum_{j=0}^{r}(-1)^{j} \bar{\pi}_{i_{j}-i_{j+1}},
$$

where $\pi_{i_{j}-i_{j+1}}$ is the orthogonal projection onto $V_{i_{j}} \cap V_{i_{j+1}}^{\perp}$ and $\bar{\pi}_{i_{j}-i_{j+1}}$ the orthogonal projection onto the corresponding conjugate space. Hence, the +1 -eigenspace of $s_{0} \exp \zeta_{I_{1}}^{\prime}$ has dimension $2 d+1$, with $d=\sum_{j=1}^{r}(-1)^{j+1} i_{j}$, which means that $P_{\zeta^{\prime}}^{\sigma_{\varrho}} \cong G_{2 d+1}\left(\mathbb{R}^{2 n}\right)$.

In particular, we have $P_{\zeta_{d}}^{\sigma_{\varrho}} \cong G_{2 d+1}\left(\mathbb{R}^{2 n}\right)$ for each $d \in\{1, \ldots, n-1\}$.

Theorem 22. Each harmonic map from $S^{2}$ into the real Grassmannian $G_{2 d+1}\left(\mathbb{R}^{2 n}\right)$ belongs to one of the following classes: $\left(\zeta, \operatorname{Ad} \exp \pi(\tilde{\zeta}-\zeta) \circ \sigma_{\varrho, l}\right)$, where $\zeta$ and $\tilde{\zeta}$ are $\varrho$-canonical elements such that $\tilde{\zeta} \preceq \zeta$ and $\tilde{\zeta}=\sum_{i \in I_{1}} \zeta_{i}+\zeta_{l}$, where

a) $I_{1}=\left\{i_{1}>i_{2}>\ldots>i_{r}\right\}$ satisfies $d=\sum_{j=1}^{r}(-1)^{j+1} i_{j}$;

b) $l \in\{0,1, \ldots, n-1\}$ and $l \notin I_{1}$ (if $l=0$, we set $\zeta_{0}=0$ ). 
Proof. We consider harmonic maps into $P_{\zeta_{d}}^{\sigma_{e}} \cong G_{2 d+1}\left(\mathbb{R}^{2 n}\right)$. Let $\zeta^{\prime}$ be a $\varrho$-semi-canonical element and write $\zeta^{\prime}=\sum_{i \in I_{1}} \zeta_{i}+\sum_{i \in I_{2}} 2 \zeta_{i}$ for some disjoint subsets $I_{1}$ and $I_{2}$ of $\{1, \ldots, n-1\}$. By Proposition 21, $P_{\zeta^{\prime}}^{\sigma_{o}} \cong$ $G_{2 d+1}\left(\mathbb{R}^{2 n}\right)$ if and only if either $d=\sum_{j=1}^{r}(-1)^{j+1} i_{j}$ or $n-d-1=\sum_{j=1}^{r}(-1)^{j+1} i_{j}$, since $G_{2 d+1}\left(\mathbb{R}^{2 n}\right)$ and $G_{2 d^{\prime}+1}\left(\mathbb{R}^{2 n}\right)$, with $d^{\prime}=n-d-1$, can be identified via $V \mapsto V^{\perp}$. However, it follows from the same reasoning as in the proof of Theorem 19 that, in the second case, $P_{\zeta^{\prime}}^{\sigma_{e}}$ does not coincide with the connected component $P_{\zeta_{d}}^{\sigma_{\varrho}}$. So we only consider the $\varrho$-semi-canonical elements $\zeta^{\prime}$ with $d=\sum_{j=1}^{r}(-1)^{j+1} i_{j}$.

Set $l=\max I_{2}$. Next we check that the pair $\zeta^{\prime} \preceq \tilde{\zeta}=\sum_{i \in I_{1}} \zeta_{i}+\zeta_{l}$ satisfies the conditions of Proposition 16 Considering the same notations we used in the proof of Theorem 19, for each $i>0$ we have

$$
\Delta_{\varrho}^{\prime} \cap \Delta_{\zeta^{\prime}}^{2 i}=\left\{L_{j}-L_{n}|2| I_{2} \cap\{j, \ldots, l\}|+| I_{1} \cap\{j, \ldots, n-1\} \mid=2 i\right\} .
$$

In particular, for $i>0$ and $\alpha=L_{j}-L_{n} \in \Delta_{\varrho}^{\prime} \cap \Delta_{\zeta^{\prime}}^{2 i}$, it is clear that $\alpha\left(\zeta^{\prime}-\tilde{\zeta}\right) / \mathrm{i} \leq 2 i-1$, and consequently

$$
\mathfrak{g}_{2 i}^{\zeta^{\prime}} \cap \mathfrak{m}_{\varrho}^{\mathbb{C}} \subset \bigoplus_{0 \leq j<2 i} \mathfrak{g}_{j}^{\zeta^{\prime}-\tilde{\zeta}}
$$

For $i>0$, we have the decomposition

$$
\mathfrak{g}_{2 i-1}^{\zeta^{\prime}} \cap \mathfrak{k}_{\varrho}^{\mathbb{C}}=\bigoplus_{\alpha \in \Delta\left(\mathfrak{k}_{e}\right) \cap \Delta_{\zeta^{\prime}}^{2 i-1}} \mathfrak{g}_{\alpha} \oplus \bigoplus_{\alpha \in \Delta_{\varrho}^{\prime} \cap \Delta_{\zeta^{\prime}}^{2 i-1}}\left(\mathfrak{g}_{\alpha} \oplus \mathfrak{g}_{\varrho(\alpha)}\right) \cap \mathfrak{k}_{\varrho}^{\mathbb{C}} .
$$

Given $\alpha \in \mathfrak{g}_{2 i-1}^{\zeta^{\prime}}$, since $\alpha\left(\zeta^{\prime}\right) / \mathrm{i}$ is odd, we must have $\alpha\left(\zeta_{j}\right) \neq 0$ for some $j \in I_{1}$. Hence $\alpha\left(\zeta^{\prime}-\tilde{\zeta}\right) / \mathrm{i}<\alpha\left(\zeta^{\prime}\right) / \mathrm{i}$ and we conclude that

$$
\mathfrak{g}_{2 i-1}^{\zeta^{\prime}} \cap \mathfrak{k}_{\varrho}^{\mathbb{C}} \subset \bigoplus_{0 \leq j<2 i-1} \mathfrak{g}_{j}^{\zeta^{\prime}-\zeta} .
$$

The statement of the theorem follows now from Propositions 15 and 16 .

Next we will study in detail the case $G_{3}\left(\mathbb{R}^{6}\right)$. Take as base point of $G_{3}\left(\mathbb{R}^{6}\right)$ the 3-dimensional real subspace $V_{0} \oplus V_{1} \oplus \bar{V}_{1}$, where $V_{1}$ is the one-dimensional isotropic subspace spanned by $\bar{u}_{1}$. This choice establishes the identification

$$
G_{3}\left(\mathbb{R}^{6}\right) \cong S O(6) / S(O(3) \times O(3))
$$

and the corresponding involution is $\sigma_{\varrho, 1}=\operatorname{Ad}\left(\exp \pi \zeta_{1}\right) \circ \sigma_{\varrho}$. Following our classification procedure, we also identify $G_{3}\left(\mathbb{R}^{6}\right)$ with $P_{\zeta_{1}}^{\sigma_{e}}$ via the totally geodesic embedding (13). From Theorem 22, we have six classes of harmonic maps into $G_{3}\left(\mathbb{R}^{6}\right)$ :

$$
\left(\zeta_{1}, \sigma_{\varrho}\right), \quad\left(\zeta_{1}+\zeta_{2}, \sigma_{\varrho}\right), \quad\left(\zeta_{2}, \sigma_{\varrho, 1}\right), \quad\left(\zeta_{1}, \sigma_{\varrho, 2}\right), \quad\left(\zeta_{1}+\zeta_{2}, \sigma_{\varrho, 2}\right), \quad\left(\zeta_{2}, \operatorname{Ad}\left(\exp \pi \zeta_{2}\right) \circ \sigma_{\varrho, 1}\right) .
$$

Theorem 23. Let $\varphi: S^{2} \rightarrow G_{3}\left(\mathbb{R}^{6}\right)$ be an harmonic map.

(1) If $\varphi$ is associated to the pair $\left(\zeta_{1}, \sigma_{\varrho}\right)$ then $\varphi$ is $S^{1}$-invariant and, up to isometry, is given by

$$
\varphi=V_{0} \oplus V \oplus \bar{V},
$$

where $V$ is a holomorphic isotropic subbundle of $S^{2} \times V_{0}^{\perp}$ of rank 1 satisfying $\partial V \subseteq \bar{V}^{\perp}$.

(2) If $\varphi$ is associated to the pair $\left(\zeta_{1}+\zeta_{2}, \sigma_{\varrho}\right)$ and is $S^{1}$-invariant, then, up to isometry,

$$
\varphi=V_{0} \oplus\left(W \cap V^{\perp}\right) \oplus\left(\overline{W \cap V^{\perp}}\right),
$$

where $V \subset W$ are holomorphic isotropic subbundles of $S^{2} \times V_{0}^{\perp}$ of rank 1 and 2, respectively, satisfying $\partial V \subset W$ and $\partial W \subset \bar{W}^{\perp}$.

(3) If $\varphi$ is associated to the pair $\left(\zeta_{2}, \sigma_{\varrho, 1}\right)$ and is $S^{1}$-invariant, then, up to isometry,

$$
\varphi=\left\{\left(L_{1} \oplus \bar{L}_{1}\right)^{\perp} \cap\left(V_{0} \oplus V_{1} \oplus \bar{V}_{1}\right)\right\} \oplus\left(L_{2} \oplus \bar{L}_{2}\right),
$$

where $L_{1}$ and $L_{2}$ are holomorphic isotropic bundle lines of $S^{2} \times\left(V_{0} \oplus V_{1} \oplus \bar{V}_{1}\right)$ and $S^{2} \times\left(V_{0} \oplus V_{1} \oplus \bar{V}_{1}\right)^{\perp}$, respectively. 
The corresponding extended solutions have uniton number 2, 4, and 2, respectively, with respect to the standard representation of $S O(6)$. The harmonic maps in the classes $\left(\zeta_{1}, \sigma_{\varrho, 2}\right),\left(\zeta_{1}+\zeta_{2}, \sigma_{\varrho, 2}\right), \operatorname{and}\left(\zeta_{2}, \operatorname{Ad}\left(\exp \pi \zeta_{2}\right) \circ\right.$ $\left.\sigma_{\varrho, 1}\right)$ are precisely the orthogonal complements of the harmonic maps in the classes $\left(\zeta_{1}, \sigma_{\varrho}\right),\left(\zeta_{1}+\zeta_{2}, \sigma_{\varrho}\right)$, and $\left(\zeta_{2}, \sigma_{\varrho, 1}\right)$, respectively.

Proof. For the first two classes, and according to our classification procedure, we identify $G_{3}\left(\mathbb{R}^{6}\right)$ with $P_{\zeta_{1}}^{\sigma_{\varrho}}$ via the totally geodesic embedding $g \cdot\left(V_{0} \oplus V_{1} \oplus \bar{V}_{1}\right) \mapsto \exp \left(\pi \zeta_{1}\right) g \sigma_{\varrho, 1}\left(g^{-1}\right)$. In these two cases, $T_{\sigma_{\varrho}}$-invariant extended solutions $\Phi$ associated to harmonic maps $\varphi=g \cdot\left(V_{0} \oplus V_{1} \oplus \bar{V}_{1}\right)$ satisfy

$$
\Phi_{-1}=\exp \left(\pi \zeta_{1}\right) g \sigma_{\varrho, 1}\left(g^{-1}\right)=\exp \left(\pi \zeta_{1}\right)\left(\pi_{\varphi}-\pi_{\varphi}^{\perp}\right) \exp \left(\pi \zeta_{1}\right) s_{0}
$$

First we consider the harmonic maps associated to the pair $\left(\zeta_{1}, \sigma_{\varrho}\right)$. We have $r\left(\zeta_{1}\right)=1$ and

$$
\left(\mathfrak{u}_{\zeta_{1}}^{0}\right)_{\sigma_{\varrho}}=\left(\mathfrak{p}_{0}^{\zeta_{1}}\right)^{\perp} \cap \mathfrak{k}_{\varrho}^{\mathbb{C}}=\mathfrak{g}_{1}^{\zeta_{1}} \cap \mathfrak{k}_{\varrho}^{\mathbb{C}} .
$$

Consequently any such harmonic map is $S^{1}$-invariant. Write $\gamma_{\zeta_{1}}(\lambda)=\lambda^{-1} \pi_{V_{1}}+\pi_{V_{1} \oplus \bar{V}_{1}}^{\perp}+\lambda \pi_{\bar{V}_{1}}$, where $V_{1}$ is the one-dimensional isotropic space spanned by $\bar{u}_{1}$. Let $\Phi: S^{2} \backslash D \rightarrow U_{\zeta_{1}}^{\sigma_{\varrho}}$ be an extended solution associated to the harmonic map $\varphi$. Then, by $S^{1}$-invariance, we can write

$$
\Phi_{\lambda}=\lambda^{-1} \pi_{V}+\pi_{V \oplus \bar{V}}^{\perp}+\lambda \pi_{\bar{V}}
$$

where $V$ is a holomorphic isotropic subbundle of $S^{2} \times \mathbb{R}^{6}$ of rank 1 satisfying $\partial V \subseteq \bar{V}^{\perp}$. The $T_{\sigma_{\rho}}$-invariance of $\Phi$ implies that $V_{0} \subset(V \oplus \bar{V})^{\perp}$. Equating (29) and (30), we get, up to isometry, $\varphi=V_{0} \oplus V \oplus \bar{V}$.

For the case $\left(\zeta_{1}+\zeta_{2}, \sigma_{\varrho}\right)$, since

$$
\gamma_{\zeta_{1}+\zeta_{2}}(\lambda)=\lambda^{-2} \pi_{V_{1}}+\lambda^{-1} \pi_{V_{2} \cap V_{1}^{\perp}}+\pi_{V_{2} \oplus \bar{V}_{2}}^{\perp}+\lambda \pi_{\bar{V}_{2} \cap \bar{V}_{1}^{\perp}}+\lambda^{2} \pi_{\bar{V}_{1}}
$$

any $S^{1}$-invariant harmonic map $\varphi$ in this class admits an extended solution of the form

$$
\Phi_{\lambda}=\lambda^{-2} \pi_{V}+\lambda^{-1} \pi_{W \cap V^{\perp}}+\pi_{W \oplus \bar{W}}^{\perp}+\lambda \pi_{\bar{W} \cap \bar{V}^{\perp}}+\lambda^{2} \pi_{\bar{V}}
$$

where $V \subset W$ are holomorphic isotropic subbundles of rank 1 and 2, respectively, satisfying $\partial V \subset W$ and $\partial W \subset \bar{W}^{\perp}$. By $T_{\sigma_{e}}$-invariance, we must have $V_{0} \subset(W \oplus \bar{W})^{\perp}$, hence $V \subset W$ are subbundles of $S^{2} \times V_{0}^{\perp}$. Equating (29) and (32), we get (27).

For the case $\left(\zeta_{2}, \sigma_{\varrho, 1}\right)$, we identify $G_{3}\left(\mathbb{R}^{6}\right)$ with $P_{\zeta_{2}}^{\sigma_{\varrho}, 1}=\exp \pi \zeta_{1} P_{\zeta_{2}-\zeta_{1}}^{\sigma_{\varrho}}$ via the totally geodesic embedding

$$
g \cdot\left(V_{0} \oplus V_{1} \oplus \bar{V}_{1}\right) \mapsto g \sigma_{\varrho, 1}\left(g^{-1}\right) .
$$

Extended solutions $\Phi$ associated to $S^{1}$-invariant harmonic maps in this class must be of the form

$$
\Phi_{\lambda}=\lambda^{-1} \pi_{W}+\pi_{W \oplus \bar{W}}^{\perp}+\lambda \pi_{W}
$$

where $W$ is a holomorphic isotropic subbundle of rank 2. By $T_{\sigma_{\varrho, 1}}$-invariance, we must have $\left[\pi_{W}, \pi_{V_{0} \oplus V_{1} \oplus \bar{V}_{1}}\right]=0$, which means that $W$ must be of the form $W=L_{1} \oplus L_{2}$, where $L_{1}$ and $L_{2}$, respectively, are holomorphic isotropic bundle lines of $S^{2} \times\left(V_{0} \oplus V_{1} \oplus \bar{V}_{1}\right)$ and $S^{2} \times\left(V_{0} \oplus V_{1} \oplus \bar{V}_{1}\right)^{\perp}$.

On the other hand, in view of (33), we have $\Phi_{-1}=\left(\pi_{\varphi}-\pi_{\varphi}^{\perp}\right) \exp \left(\pi \zeta_{1}\right) s_{0}$. Equating this with (34), we conclude that (28) holds. The remaining cases are treated similarly.

Remark 5. The first two classes of $S^{1}$-invariant harmonic maps $\varphi: S^{2} \rightarrow G_{3}\left(\mathbb{R}^{6}\right)$ in Theorem 23 factor through $G_{2}\left(\mathbb{R}^{5}\right)$. That is, for any such harmonic map $\varphi$, there exists $\tilde{\varphi}: S^{2} \rightarrow G_{2}\left(\mathbb{R}^{5}\right)$, where we identify $\mathbb{R}^{5}$ with $V_{0}^{\perp}$, such that $\varphi=V_{0} \oplus \tilde{\varphi}$. An explicit construction of all harmonic maps from $S^{2}$ into $G_{2}\left(\mathbb{R}^{n}\right)$ can be found in $[16]$. In that paper, harmonic maps of the form (26) are called real mixed pairs. We emphasise that the harmonic maps into $G_{3}\left(\mathbb{R}^{6}\right)$ associated to extended solutions in the corresponding unstable manifolds need not to factor through $G_{2}\left(\mathbb{R}^{5}\right)$ in the same way. 
Let us consider the case $\left(\zeta_{1}+\zeta_{2}, \sigma_{\varrho}\right)$. Taking into account the Weierstrass representation of Theorem 17 any extended solution $\Phi: S^{2} \backslash D \rightarrow U_{\zeta}^{\sigma_{\varrho}}(S O(6))$, with $\zeta=\zeta_{1}+\zeta_{2}$, can be written as $\Phi=\exp C \cdot \gamma_{\zeta}$, for some meromorphic vector-valued function $C: S^{2} \rightarrow\left(\mathfrak{u}_{\zeta}^{0}\right)_{\sigma_{\varrho}}$. We have $r(\zeta)=3$ and

$$
\left(\mathfrak{u}_{\zeta}^{0}\right)_{\sigma_{\varrho}}=\left(\mathfrak{g}_{1}^{\zeta} \oplus \mathfrak{g}_{2}^{\zeta} \oplus \mathfrak{g}_{3}^{\zeta}\right) \cap \mathfrak{k}_{\varrho}^{\mathbb{C}} \oplus \lambda\left(\mathfrak{g}_{2}^{\zeta} \oplus \mathfrak{g}_{3}^{\zeta}\right) \cap \mathfrak{m}_{\varrho}^{\mathbb{C}} \oplus \lambda^{2} \mathfrak{g}_{3}^{\zeta} \cap \mathfrak{k}_{\varrho}^{\mathbb{C}} .
$$

Moreover,

$$
\begin{gathered}
\mathfrak{g}_{1}^{\zeta} \cap \mathfrak{k}_{\varrho}^{\mathbb{C}}=\mathfrak{g}_{L_{1}-L_{2}} \oplus\left\{\left(\mathfrak{g}_{L_{2}-L_{3}} \oplus \mathfrak{g}_{L_{2}+L_{3}}\right) \cap \mathfrak{k}_{\varrho}^{\mathbb{C}}\right\}, \quad \mathfrak{g}_{2}^{\zeta} \cap \mathfrak{k}_{\varrho}^{\mathbb{C}}=\left(\mathfrak{g}_{L_{1}+L_{3}} \oplus \mathfrak{g}_{L_{1}-L_{3}}\right) \cap \mathfrak{k}_{\varrho}^{\mathbb{C}}, \\
\mathfrak{g}_{3}^{\zeta} \cap \mathfrak{k}_{\varrho}^{\mathbb{C}}=\mathfrak{g}_{L_{1}+L_{2}}, \quad\left(\mathfrak{g}_{2}^{\zeta} \oplus \mathfrak{g}_{3}^{\zeta}\right) \cap \mathfrak{m}_{\varrho}^{\mathbb{C}}=\mathfrak{g}_{2}^{\zeta} \cap \mathfrak{m}_{\varrho}^{\mathbb{C}}=\left(\mathfrak{g}_{L_{1}-L_{3}} \oplus \mathfrak{g}_{L_{1}+L_{3}}\right) \cap \mathfrak{m}_{\varrho}^{\mathbb{C}} .
\end{gathered}
$$

Write

$$
C=C_{0}+\lambda C_{1}+\lambda^{2} C_{2}, \quad C_{0}=c_{0}^{1}+c_{0}^{2}+c_{0}^{3}, \quad C_{1}=c_{1}^{2}+c_{1}^{3}, \quad C_{2}=c_{2}^{3}
$$

where the functions $c_{0}^{i}: S^{2} \rightarrow \mathfrak{g}_{i}^{\zeta} \cap \mathfrak{k}_{\varrho}^{\mathbb{C}}, c_{1}^{i}: S^{2} \rightarrow \mathfrak{g}_{i}^{\zeta} \cap \mathfrak{m}_{\varrho}^{\mathbb{C}}$, and $c_{2}^{3}: S^{2} \rightarrow \mathfrak{g}_{3}^{\zeta} \cap \mathfrak{k}_{\varrho}^{\mathbb{C}}$ are meromorphic functions. Clearly, $c_{1}^{3}=0$. Consider the root vectors defined by (17). Since $\sigma_{\varrho}\left(X_{2,3}\right)=-Y_{2,3}$ and $\sigma_{\varrho}\left(X_{1,3}\right)=-Y_{1,3}$, we can write

$$
c_{0}^{1}=a X_{1,2}+b\left(X_{2,3}-Y_{2,3}\right), c_{0}^{2}=c\left(X_{1,3}-Y_{1,3}\right), c_{0}^{3}=d Y_{1,2}, c_{1}^{2}=e\left(X_{1,3}+Y_{1,3}\right), c_{2}^{3}=f X_{1,2}
$$

in terms of $\mathbb{C}$-valued meromorphic functions $a, b, c, d, e, f$.

Taking into account the results of Section 3.2 $\Phi=\exp C \cdot \gamma_{\zeta}$ is an extended solution if and only if, in the expression

we have:

$$
(\exp C)^{-1}(\exp C)_{z}=C_{z}-\frac{1}{2 !}(\operatorname{ad} C) C_{z}+\frac{1}{3 !}(\operatorname{ad} C)^{2} C_{z}
$$

a) the independent coefficient should have zero component in each $\mathfrak{g}_{2}^{\zeta}$ and $\mathfrak{g}_{3}^{\zeta}$, that is

$$
c_{0 z}^{2}-\frac{1}{2}\left[c_{0}^{1}, c_{0 z}^{1}\right]=0, \quad c_{0 z}^{3}-\frac{1}{2}\left[c_{0}^{1}, c_{0 z}^{2}\right]-\frac{1}{2}\left[c_{0}^{2}, c_{0 z}^{1}\right]+\frac{1}{6}\left[c_{0}^{1},\left[c_{0}^{1}, c_{0 z}^{1}\right]\right]=0 ;
$$

b) the $\lambda$ coefficient should have zero component in $\mathfrak{g}_{3}^{\zeta}$, that is

$$
\left[c_{0}^{1}, c_{1 z}^{2}\right]+\left[c_{1}^{2}, c_{0 z}^{1}\right]=0 .
$$

From equations (36) we get the equations (prime denotes $z$-derivative)

$$
2 c^{\prime}=a b^{\prime}-b a^{\prime}, \quad 3 d^{\prime}=3 c b^{\prime}-b c^{\prime} ;
$$

on the other hand, observe that (37) always holds since

$$
\left[c_{0}^{1}, c_{1 z}^{2}\right]+\left[c_{1}^{2}, c_{0 z}^{1}\right] \in\left[\mathfrak{g}_{1}^{\zeta} \cap \mathfrak{k}_{\varrho}^{\mathbb{C}}, \mathfrak{g}_{2}^{\zeta} \cap \mathfrak{m}_{\varrho}^{\mathbb{C}}\right] \subset \mathfrak{g}_{3}^{\zeta} \cap \mathfrak{m}_{\varrho}^{\mathbb{C}}=\{0\} .
$$

Hence we conclude that, any extended solution $\Phi: S^{2} \backslash D \rightarrow U_{\zeta}^{\sigma_{\varrho}}(S O(6))$, with $\zeta=\zeta_{1}+\zeta_{2}$, of the form $\Phi=\exp C \cdot \gamma_{\zeta}$, can be constructed as follows: choose arbitrary meromorphic functions $a, b, e$ and $f$; integrate equations (38) to obtain the meromorphic functions $c$ and $d ; C$ is then given by (35).

Example 3. Choose $a(z)=b(z)=z$. From (38), we can take $c(z)=1$ and $d(z)=z$. This data defines the matrix $C_{0}$ and the $S^{1}$-invariant extended solution $\Phi^{0}=\exp C_{0} \cdot \gamma_{\zeta}$, where the loop $\gamma_{\zeta}$, with $\zeta=\zeta_{1}+\zeta_{2}$, is given by (31). The extended solutions $\Phi: S^{2} \rightarrow U_{\zeta}^{\sigma_{\varrho}}(S O(6))$ satisfying $\Phi^{0}=u_{\zeta} \circ \Phi$ are of the form $\Phi=\exp C \cdot \gamma_{\zeta}$, where the matrix $C=C_{0}+C_{1} \lambda+C_{2} \lambda^{2}$ is given by

$$
C=\left(\begin{array}{cccccc}
0 & z & 1 & 0 & z & -1 \\
0 & 0 & z & -z & 0 & -z \\
0 & 0 & 0 & 1 & z & 0 \\
0 & 0 & 0 & 0 & 0 & 0 \\
0 & 0 & 0 & -z & 0 & 0 \\
0 & 0 & 0 & -1 & -z & 0
\end{array}\right)+\left(\begin{array}{cccccc}
0 & 0 & e \lambda & 0 & f \lambda^{2} & -e \lambda \\
0 & 0 & 0 & -f \lambda^{2} & 0 & 0 \\
0 & 0 & 0 & e \lambda & 0 & 0 \\
0 & 0 & 0 & 0 & 0 & 0 \\
0 & 0 & 0 & 0 & 0 & 0 \\
0 & 0 & 0 & -e \lambda & 0 & 0
\end{array}\right)
$$


with respect to the complex orthonormal basis $\mathbf{u}=\left\{u_{1}, u_{2}, u_{3}, \bar{u}_{1}, \bar{u}_{2}, \bar{u}_{3}\right\}$, where $e$ and $f$ are arbitrary meromorphic functions on $S^{2}$. The holomorphic vector bundles $V$ and $W$ associated to the $S^{1}$-invariant extended solution $\exp C_{0} \cdot \gamma_{\zeta}$ are given by $V=\exp C_{0} \cdot V_{1}$ and $W=\exp C_{0} \cdot V_{2}$, and we have, with respect to the basis $\mathbf{u}$,

$$
\begin{aligned}
V & =\operatorname{span}\left\{\left(12-12 z^{2}-z^{4},-4 z^{3}, 12-6 z^{2}, 12,-12 z,-12+6 z^{2}\right)\right\} \\
W & =\operatorname{span}\left\{\left(6 z+z^{3}, 3 z^{2}, 3 z, 0,3,-3 z\right)\right\} \oplus V .
\end{aligned}
$$

5.2. Outer symmetric $S U(2 n+1)$-spaces. Let $E_{j}$ be the square $(m \times m)$-matrix whose $(j, j)$-entry is i and all other entries are 0 . The complexification $\mathfrak{t}^{\mathbb{C}}$ of the algebra $\mathfrak{t}$ of diagonal matrices $\sum a_{i} E_{i}$, with $a_{i} \in \mathbb{C}$ and $\sum a_{i}=0$, is a Cartan subalgebra of $\mathfrak{s u}(m)^{\mathbb{C}}$. Let $\left\{L_{1}, \ldots, L_{m}\right\}$ be the dual basis of $\left\{E_{1}, \ldots, E_{m}\right\}$, that is $L_{i}\left(E_{j}\right)=\mathrm{i} \delta_{i j}$. The roots of $\mathfrak{s u}(m)$ are the vectors $L_{i}-L_{j}$, with $i \neq j$ and $1 \leq i, j \leq m-1$ and $\Delta^{+}=\left\{L_{i}-L_{j}\right\}_{i<j}$ is a positive root system with positive simple roots $\alpha_{i}=L_{i}-L_{i+1}$, for $1 \leq i \leq m-1$. For $i \neq j$, the matrix $X_{i, j}$ whose $(i, j)$ entry is 1 and all other entries are 0 generate the root space $\mathfrak{g}_{L_{i}-L_{j}}$. The dual basis of $\Delta_{0}=\left\{\alpha_{1}, \ldots, \alpha_{m-1}\right\}$ in it* is formed by the matrices

$$
H_{i}=\frac{m-i}{m}\left(E_{1}+\ldots+E_{i}\right)-\frac{i}{m}\left(E_{i+1}+\ldots+E_{m}\right) .
$$

5.2.1. Special Lagrangian spaces. Consider on $\mathbb{R}^{2 m}$ the standard inner product $\langle\cdot, \cdot\rangle$ and the canonical orthonormal basis $\mathbf{e}^{2 m}=\left\{e_{1}, \ldots, e_{2 m}\right\}$. Define the orthogonal complex structure $I$ by $I\left(e_{i}\right)=e_{2 m+1-i}$, for $i \in\{1, \ldots, m\}$. A Lagrangian subspace of $\mathbb{R}^{2 m}$ (with respect to $I$ ) is a $m$-dimensional subspace $L$ such that $I L \perp L$. Let $\mathcal{L}_{m}$ be the space of all Lagrangian subspaces of $\mathbb{R}^{2 m}$ and $L_{0} \in \mathcal{L}_{m}$ the Lagrangian subspace generated by $\mathbf{e}^{m}=\left\{e_{1}, \ldots, e_{m}\right\}$. The unitary group $U(m)$ acts transitively on $\mathcal{L}_{m}$, with isotropy group at $L_{0}$ equal to $S O(m)$, and $\mathcal{L}_{m}$ is a reducible symmetric space that can be identified with $U(m) / S O(m)$ (see [18] for details).

The space $\mathcal{L}_{m}$ can also be interpreted as the set of all orthogonal linear maps $\tau: \mathbb{R}^{2 m} \rightarrow \mathbb{R}^{2 m}$ satisfying $\tau^{2}=e$ and $I \tau=-\tau I$. Indeed, if $V_{ \pm}$are the \pm 1 eigenspaces of $\tau$, then $I V_{+}=V_{-}$and $I V_{+} \perp V_{+}$, that is $V_{+}$ is Lagrangian. From this point of view, $U(m)$ acts on $\mathcal{L}_{m}$ by conjugation: $g \cdot \tau=g \tau g^{-1}$. Let $\tau_{0} \in \mathcal{L}_{m}$ be the orthogonal linear map corresponding to $L_{0}$, that is, $\tau_{\left.0\right|_{L_{0}}}=e$ and $\tau_{\left.0\right|_{I L_{0}}}=-e$. The corresponding involution on $U(m)$ is given by $\sigma(g)=\tau_{0} g \tau_{0}$ and the Cartan embedding $\iota: \mathcal{L}_{m} \hookrightarrow U(m)$ is given by $\iota(\tau)=\tau \tau_{0}$.

The totally geodesic submanifold $\mathcal{L}_{m}^{s}:=S U(m) / S O(m)$ of $U(m) / S O(m)$ is also known as the space of special Lagrangian subspaces of $\mathbb{R}^{2 m}$. It is an irreducible outer symmetric $S U(m)$-space.

5.2.2. Harmonic maps into $\mathcal{L}_{2 n+1}^{s}$. Take $m=2 n+1$. The non-trivial involution $\varrho$ of the Dynkin diagram of $\mathfrak{s u}(2 n+1)^{\mathbb{C}}$ is given by $\varrho\left(\alpha_{i}\right)=\alpha_{2 n+1-i}$. In particular, $\varrho$ does not fix any root in $\Delta_{0}$ and there exists only one class of outer symmetric $S U(2 n+1)$-spaces. The semi-fundamental basis $\pi_{\mathfrak{k}_{e}}\left(\Delta_{0}\right)=\left\{\beta_{1}, \ldots, \beta_{n}\right\}$ is given by $\beta_{i}=\frac{1}{2}\left(\alpha_{i}+\alpha_{2 n+1-i}\right)$ whereas the dual basis $\left\{\zeta_{1}, \ldots, \zeta_{n}\right\}$ is given by

$$
\zeta_{i}=H_{i}+H_{2 n+1-i}=E_{1}+\ldots+E_{i}-\left(E_{2 n+2-i}+\ldots+E_{2 n+1}\right),
$$

for $1 \leq i \leq n$. Since each $\zeta_{i}$ belongs to the integer lattice $\mathfrak{I}(S U(2 n+1))$, the $\varrho$-semi-canonical elements of $S U(2 n+1)$ are precisely the elements $\zeta=\sum_{i=1}^{n} m_{i} \zeta_{i}$ with $m_{i} \in\{0,1,2\}$.

Let $\mathbf{e}^{2 n+1}=\left\{e_{1}, \ldots, e_{2 n+1}\right\}$ be the canonical orthonormal basis of $\mathbb{R}^{2 n+1}$. Identify $\mathbb{C}^{2 n+1}$ with $\left(\mathbb{R}^{4 n+2}, I\right)$, where $I$ is defined as above. Set

$$
v_{j}=\frac{1}{\sqrt{2}}\left(e_{j}+\mathrm{i} e_{2 n+2-j}\right)
$$

for $1 \leq j \leq n, v_{n+1}=e_{n+1}$ and $v_{2 n+2-j}=\bar{v}_{j}$. Take the matrices $E_{j}$ with respect to the complex basis $\mathbf{v}=\left\{v_{1}, \ldots, v_{2 n+1}\right\}$ of $\mathbb{C}^{2 n+1}$. Hence $\tau_{0} E_{j} \tau_{0}=-E_{2 n+2-j}$ and the fundamental involution $\sigma_{\varrho}$ is given by $\sigma_{\varrho}(g)=\tau_{0} g \tau_{0}$. The fundamental outer symmetric $S U(2 n+1)$-space is the space of special Lagrangian subspaces $\mathcal{L}_{2 n+1}^{s}=S U(2 n+1) / S O(2 n+1)$, and this is the unique outer symmetric $S U(2 n+1)$-space.

Next we consider in detail harmonic maps into $\mathcal{L}_{3}^{s}$. In this case we have two non-zero $\varrho$-semi-canonical elements, $\zeta_{1}$ and $2 \zeta_{1}$, and consequently two classes of harmonic maps, $\left(\zeta_{1}, \sigma_{\varrho}\right)$ and $\left(\zeta_{1}, \sigma_{\varrho, 1}\right)$. Since $\zeta_{1}=E_{1}-E_{3}$, 
we have $r\left(\zeta_{1}\right)=\left(L_{1}-L_{3}\right)\left(\zeta_{1}\right) / \mathrm{i}=2$. Let $W_{1}, W_{2}$ and $W_{3}$ be the complex one-dimensional images of $E_{1}, E_{2}$ and $E_{3}$, respectively. Any extended solution

$$
\Phi: S^{2} \backslash D \rightarrow U_{\zeta_{1}}^{\sigma_{\varrho}}(S U(2 n+1))
$$

is given by $\Phi=\exp C \cdot \gamma_{\zeta_{1}}$, with $\gamma_{\zeta_{1}}(\lambda)=\lambda^{-1} \pi_{W_{3}}+\pi_{W_{2}}+\lambda \pi_{W_{1}}$, for some holomorphic vector-valued function $C: S^{2} \backslash D \rightarrow\left(\mathfrak{u}_{\zeta_{1}}^{0}\right)_{\sigma_{\varrho}}$, where

$$
\left(\mathfrak{u}_{\zeta_{1}}^{0}\right)_{\sigma_{\varrho}}=\left(\mathfrak{p}_{0}^{\zeta_{1}}\right)^{\perp} \cap \mathfrak{k}_{\varrho}^{\mathbb{C}}+\lambda\left(\mathfrak{p}_{1}^{\zeta_{1}}\right)^{\perp} \cap \mathfrak{m}_{\varrho}^{\mathbb{C}}
$$

and

$$
\left(\mathfrak{p}_{0}^{\zeta_{1}}\right)^{\perp} \cap \mathfrak{k}_{\varrho}^{\mathbb{C}}=\left(\mathfrak{g}_{L_{1}-L_{2}} \oplus \mathfrak{g}_{L_{2}-L_{3}} \oplus \mathfrak{g}_{L_{1}-L_{3}}\right) \cap \mathfrak{k}_{\varrho}^{\mathbb{C}}, \quad\left(\mathfrak{p}_{1}^{\zeta_{1}}\right)^{\perp} \cap \mathfrak{m}_{\varrho}^{\mathbb{C}}=\mathfrak{g}_{L_{1}-L_{3}} \cap \mathfrak{m}_{\varrho}^{\mathbb{C}}
$$

Let $X_{i, j}$ be the square matrix whose $(i, j)$ entry is 1 and all the other entries are 0 , with respect to the basis $\mathbf{v}$. The root space $\mathfrak{g}_{L_{i}-L_{j}}$ is spanned by $X_{i, j}$. We have $\sigma_{\varrho}\left(X_{1,2}\right)=-X_{2,3}$ and $\sigma_{\varrho}\left(X_{1,3}\right)=-X_{1,3}$ (consequently, $\left.\mathfrak{g}_{L_{1}-L_{3}} \subset \mathfrak{m}_{\varrho}^{\mathbb{C}}\right)$. Hence we can write $C=C_{0}+C_{1} \lambda$, with $C_{0}=a\left(X_{1,2}-X_{2,3}\right)$ and $C_{1}=b X_{1,3}$, for some meromorphic functions $a, b$ on $S^{2}$. The harmonicity equations do not impose any condition on these meromorphic functions, hence any harmonic map $\varphi: S^{2} \rightarrow \mathcal{L}_{3}^{s}$ in the class $\left(\zeta_{1}, \sigma_{\varrho}\right)$ admits an extended solution of the form

$$
\Phi=\exp \left(\begin{array}{ccc}
0 & a & b \lambda \\
0 & 0 & -a \\
0 & 0 & 0
\end{array}\right) \cdot \gamma_{\zeta_{1}}=\left(\begin{array}{ccc}
1 & a & \frac{1}{2}\left(-a^{2}+2 b \lambda\right) \\
0 & 1 & -a \\
0 & 0 & 1
\end{array}\right) \cdot \gamma_{\zeta_{1}},
$$

and $\varphi$ is recovered by setting $\varphi=\Phi_{-1} \tau_{0}$. Similarly, one can see that the class of harmonic maps in $\left(\zeta_{1}, \sigma_{\varrho, 1}\right)$ admits an extended solution of the form

$$
\Phi=\left(\begin{array}{ccc}
1 & a & \frac{1}{2}\left(a^{2}+2 b \lambda\right) \\
0 & 1 & a \\
0 & 0 & 1
\end{array}\right) \cdot \gamma_{\zeta_{1}}
$$

with no restrictions on the meromorphic functions $a$ and $b$.

H. Ma established (cf. Theorem 4.1 of [13]) that harmonic maps $\varphi: S^{2} \rightarrow \mathcal{L}_{3}^{s}$ are essentially of two types: 1) $\iota_{\sigma} \circ \varphi$ is a Grassmannian solution obtained from a full harmonic map $f: S^{2} \rightarrow \mathbb{R} P^{2} \subset \mathbb{C} P^{2}$, where $\iota_{\sigma}$ is the Cartan embedding of $\mathcal{L}_{3}^{s}$ in $\left.S U(3) ; 2\right)$ up to left multiplication by a constant, $\iota_{\sigma} \circ \varphi$ is of the form $\left(\pi_{\beta_{1}}-\pi_{\beta_{1}}^{\perp}\right)\left(\pi_{\beta_{2}}-\pi_{\beta_{2}}^{\perp}\right)$, where $\beta_{1}$ is a Frenet pair associated to a full totally istotropic holomorphic map $g: S^{2} \rightarrow \mathbb{C} P^{2}$ and $\beta_{2}$ is a rank 1 holomorphic subbundle of $G^{\prime}(g)^{\perp}$, where $G^{\prime}(g)$ is the first Gauss bundle of $g$. Observe that if, in the second case, $\beta_{2}$ coincides with $g$, then $\iota_{\sigma} \circ \varphi$ is a Grassmannian solution obtained from the full harmonic map $f:=G^{\prime}(g)$ from $S^{2}$ to $\mathbb{R} P^{2}$, that is, $\varphi$ is of type 1). Comparing this with our description, it is not difficult to see that harmonic maps of type 1) are $S^{1}$-invariant extended solutions (take $b=0$ in (39) and (40) and harmonic maps of type 2) are associated to extended solutions with values in the corresponding unstable manifolds (which corresponds to an arbitrary choice of $b$ in (39) and (40)). H. Ma also established a purely algebraic explicit construction of such harmonic maps in terms of meromorphic data on $S^{2}$, which is consistent with our results.

5.3. Outer symmetric $S U(2 n)$-spaces. With the same notations of Section 5.2 , the non-trivial involution $\varrho$ of the Dynkin diagram of $\mathfrak{s u}(2 n)$ is given by $\varrho\left(\alpha_{i}\right)=\alpha_{2 n-i}$, and $\varrho$ fixes the root $\alpha_{n}$. The semi-fundamental basis $\pi_{\mathfrak{k}_{e}}\left(\Delta_{0}\right)=\left\{\beta_{1}, \ldots, \beta_{n-1}\right\}$ is given by $\beta_{1}=\alpha_{n}$ and $\beta_{i}=\frac{1}{2}\left(\alpha_{i}+\alpha_{2 n-i}\right)$ if $i \geq 2$; whereas its dual basis $\left\{\zeta_{1}, \ldots, \zeta_{n-1}\right\}$ is given by

$$
\begin{aligned}
& \zeta_{1}=H_{n}=\frac{1}{2}\left(E_{1}+\ldots+E_{n}\right)-\frac{1}{2}\left(E_{n+1}+\ldots+E_{2 n}\right) \\
& \zeta_{i}=H_{i-1}+H_{2 n-i+1}=E_{1}+\ldots+E_{i-1}-\left(E_{2 n+2-i}+\ldots+E_{2 n}\right), \text { for } 2 \leq i \leq n-1 .
\end{aligned}
$$


By Theorem 7] there exist two conjugacy classes of outer involutions: the fundamental outer involution $\sigma_{\varrho}$ and $\sigma_{\varrho, 1}$. These outer involutions correspond to the symmetric spaces $S U(2 n) / S p(n)$ and $S U(2 n) / S O(2 n)$, respectively. Observe that $\zeta_{1}$ does not belong to the integer lattice $\mathfrak{I}^{\prime}\left(S U(2 n)^{\sigma_{\varrho}}\right)$ since $\exp 2 \pi \zeta_{1}=-e$.

5.3.1. Harmonic maps into the space of special unitary quaternionic structures on $\mathbb{C}^{2 n}$. A unitary quaterninonic structure on the standard hermitian space $\left(\mathbb{C}^{2 n},\langle\cdot, \cdot\rangle\right)$ is a conjugate linear map $J: \mathbb{C}^{2 n} \rightarrow \mathbb{C}^{2 n}$ satisfying $J^{2}=-I d$ and $\langle v, w\rangle=\langle J w, J v\rangle$ for all $v, w \in \mathbb{C}^{2 n}$. Consider as base point the quaternionic structure $J_{o}$ defined by $J_{o}\left(e_{i}\right)=e_{2 n+1-i}$ for each $1 \leq i \leq n$, where $\mathbf{e}^{2 n}=\left\{e_{1}, \ldots, e_{2 n}\right\}$ is the canonical hermitian basis of $\mathbb{C}^{2 n}$. The unitary group $U(2 n)$ acts transitively on the space of unitary quaternionic structures on $\mathbb{C}^{2 n}$ with isotropy group at $J_{o}$ equal to $S p(n)$, and thus $M=U(2 n) / S p(n)$. This is a reducible symmetric space with involution $\sigma: U(2 n) \rightarrow U(2 n)$ given by $\sigma(X)=J_{o} X J_{o}^{-1}$, but the totally geodesic submanifold $\mathcal{Q}_{n}^{s}:=S U(2 n) / S p(n)$ is an irreducible symmetric space, which we call the space of special unitary quaternionic structures on $\mathbb{C}^{2 n}$ (see 18 for details). If we consider the matrices $E_{i}$ with respect to the complex basis $\mathbf{v}=\left\{v_{1}, \ldots, v_{2 n}\right\}$ defined by

$$
v_{j}=\frac{1}{\sqrt{2}}\left(e_{j}+\mathrm{i} e_{2 n+1-j}\right)
$$

for $1 \leq j \leq n$, and $v_{2 n+1-j}=\bar{v}_{j}$, we see that $J_{o} E_{j} J_{o}^{-1}=-E_{2 n+1-j}$, and consequently we have $\sigma=\sigma_{\varrho}$.

Next we consider with detail harmonic maps into $\mathcal{Q}_{2}^{s}$.

Proposition 24. Each harmonic map $\varphi: S^{2} \rightarrow \mathcal{Q}_{2}^{s}$ belongs to one of the following classes: $\left(2 \zeta_{1}, \sigma_{\varrho}\right)$, and $\left(\zeta_{2}, \sigma_{\varrho, 2}\right)$.

Proof. We start by identifying $\mathcal{Q}_{2}^{s}$ with $P_{e}^{\sigma_{\varrho}}$.

The $\varrho$-semi-canonical elements of $S U(4)$ are precisely the elements

$$
2 \zeta_{1}, 4 \zeta_{1}, \zeta_{2}, 2 \zeta_{2}, 2 \zeta_{1}+\zeta_{2}, 2 \zeta_{1}+2 \zeta_{2}, 4 \zeta_{1}+\zeta_{2}, 4 \zeta_{1}+2 \zeta_{2}
$$

By Proposition 9, all these elements correspond to the symmetric space $\mathcal{Q}_{2}^{s}$.

We claim that $\exp \pi \zeta_{2}$ is not in the connected component

$$
P_{e}^{\sigma_{\varrho}}=\left\{g J_{o} g^{-1} J_{o}^{-1} \mid g \in S U(4)\right\} .
$$

In fact, $\exp \left(\pi \zeta_{2}\right) J_{o}=g J_{o} g^{-1} \cong g S p(n)$ for the unitary transformation $g$ defined by $g\left(e_{1}\right)=e_{4}, g\left(e_{4}\right)=e_{1}$, $g\left(e_{2}\right)=e_{3}$ and $g\left(e_{3}\right)=-e_{2}$. Since $\operatorname{det} g \neq 1$ we conclude that $\exp \pi \zeta_{2}$ does not belong to $P_{e}^{\sigma}$. Similarly, one can check that $\exp \pi\left(2 \zeta_{1}+\zeta_{2}\right)$ is not in $P_{e}^{\sigma_{Q}}$.

Hence, since $\exp \pi 2 \zeta_{1}$ belongs to the centre of $S U(4)$, any harmonic map $\varphi: S^{2} \rightarrow \mathcal{Q}_{2}^{s} \cong P_{e}^{\sigma_{\varrho}}$ belongs to one of the following classes: $\left(2 \zeta_{1}, \sigma_{\varrho}\right),\left(\zeta_{2}, \sigma_{\varrho, 2}\right)$, and $\left(2 \zeta_{1}+\zeta_{2}, \sigma_{\varrho, 2}\right)$. It remains to check that, in view of Proposition 16. harmonic maps in the class $\left(2 \zeta_{1}+\zeta_{2}, \sigma_{\varrho, 2}\right)$ can be normalized to harmonic maps in the class $\left(\zeta_{2}, \sigma_{\varrho, 2}\right)$.

It is clear that $2 \zeta_{1}+\zeta_{2} \preceq \zeta_{2}$. On the other hand, for any positive root $L_{i}-L_{j} \in \Delta^{+}$, with $i<j$, we have $\left(L_{i}-L_{j}\right)\left(2 \zeta_{1}\right) / \mathrm{i} \leq\left(L_{i}-L_{j}\right)\left(2 \zeta_{1}+\zeta_{2}\right) / \mathrm{i}$, where the equality holds in just one case: $\left(L_{2}-L_{3}\right)\left(2 \zeta_{1}\right)=$ $\left(L_{2}-L_{3}\right)\left(2 \zeta_{1}+\zeta_{2}\right)=2$ i. However, $\mathfrak{g}_{L_{2}-L_{3}} \subset \mathfrak{k}_{\sigma_{\varrho}, 2}$, which means that the conditions of Proposition 16$]$ hold for $\zeta=2 \zeta_{1}+\zeta_{2}$ and $\zeta^{\prime}=\zeta_{2}$, and consequently harmonic maps in the class $\left(2 \zeta_{1}+\zeta_{2}, \sigma_{\varrho, 2}\right)$ can be normalized to harmonic maps in the class $\left(\zeta_{2}, \sigma_{\varrho, 2}\right)$.

Following the same procedure as before, one can see that any harmonic map $\varphi \rightarrow \mathcal{Q}_{2}^{s}$ in the class $\left(2 \zeta_{1}, \sigma_{\varrho}\right)$ admits an extended solution of the form

$$
\Phi=\left(\begin{array}{cccc}
1 & 0 & c_{1}+a \lambda & c_{2} \\
0 & 1 & c_{3} & c_{1}-a \lambda \\
0 & 0 & 1 & 0 \\
0 & 0 & 0 & 1
\end{array}\right) \cdot \gamma_{2 \zeta_{1}}
$$

where $c_{1}, c_{2}, c_{3} \in \mathbb{C}$ are constants, $a$ is a meromorphic function on $S^{2}$. The harmonic map is recovered by setting $\varphi=\Phi_{-1} J_{o}$. Reciprocally, given arbitrary complex constants $c_{1}, c_{2}, c_{3}$ and a meromorphic function $a: S^{2} \rightarrow \mathbb{C}$ 
such $\Phi$ is an extended solution associated to some harmonic map in the class $\left(2 \zeta_{1}, \sigma_{\varrho}\right)$ (the harmonicity equations do not impose any restriction to $a$ ).

Similarly, any harmonic map $\varphi \rightarrow \mathcal{Q}_{2}^{s}$ in the class $\left(\zeta_{2}, \sigma_{\varrho, 2}\right)$ admits an extended solution of the form

$$
\Phi=\left(\begin{array}{cccc}
1 & b & a & c \\
0 & 1 & 0 & a \\
0 & 0 & 1 & -b \\
0 & 0 & 0 & 1
\end{array}\right) \cdot \gamma_{\zeta_{2}},
$$

where $a, b$ and $c$ are meromorphic functions satisfying $c^{\prime}=b a^{\prime}-b^{\prime} a$. Since $P_{\zeta_{2}, 2}^{\sigma_{Q}}=\exp \left(\pi \zeta_{2}\right) P_{e}^{\sigma_{Q}}$, the harmonic map is recovered by setting $\varphi=\exp \left(\pi \zeta_{2}\right) \Phi_{-1} J_{o}$.

5.3.2. Harmonic maps into $\mathcal{L}_{2 n}^{s}$. The outer symmetric $S U(2 n)$-space that corresponds to the involution $\sigma_{\varrho, 1}$ is the space of special Lagrangian subspaces $\mathcal{L}_{2 n}^{s} \cong S U(2 n) / S O(2 n)$. Take as base point the Lagrangian space $L_{o}=\operatorname{Span}\left\{e_{1}, \ldots, e_{2 n}\right\}$ of $\mathbb{R}^{4 n}$ and let $\tau_{0}$ be the corresponding conjugation, so that the Cartan embedding of $\mathcal{L}_{2 n}^{s}$ into $S U(2 n)$ is given by $\tau=g \tau_{o} g^{-1} \mapsto g \tau_{0} g^{-1} \tau \in P_{e}^{\sigma_{e, 1}}$.

Lemma 25. For each $\zeta \in \mathfrak{I}\left(S U(2 n)^{\sigma_{Q, 1}}\right)$ we have $\exp \pi \zeta \in P_{e}^{\sigma_{\varrho, 1}}$.

Proof. Each $\zeta \in \mathfrak{I}\left(S U(2 n)^{\sigma_{Q, 1}}\right)$ can be written as $\zeta=\sum_{i=1}^{n} n_{i}\left(E_{i}-E_{2 n+1-i}\right)$. Hence, $\exp \pi \zeta=\pi_{V}-\pi_{V}^{\perp}$, where $V=\bigoplus_{n_{i} \text { even }} \operatorname{Span}\left\{e_{i}, e_{2 n+1-i}\right\}$. Define $g \in S U(2 n)$ as follows: if $n_{i}$ is even, then $g\left(e_{i}\right)=e_{i}$ and $g\left(e_{2 n+1-i}\right)=e_{2 n+1-i}$; if $n_{i}$ is odd, then $g\left(e_{i}\right)=\mathrm{i} e_{i}$ and $g\left(e_{2 n+1-i}\right)=-\mathrm{i} e_{2 n+1-i}$. We have $\exp \pi \zeta=g \tau_{0} g^{-1} \tau_{0}$, that is $\exp \pi \zeta \in P_{e}^{\sigma_{e, 1}}$.

Now, identify $\mathcal{L}_{2 n}^{s}$ with $P_{e}^{\sigma_{\varrho, 1}}$ via its Cartan embedding. By Theorem 12, any harmonic map $\varphi: S^{2} \rightarrow P_{e}^{\sigma_{Q, 1}}$ admits an extended solution $\Phi: S^{2} \backslash D \rightarrow U_{\zeta^{\prime}}^{\sigma_{\ell, 1}}(S U(2 n))$, for some $\zeta^{\prime} \in \mathfrak{I}^{\prime}(S U(2 n)) \cap \mathfrak{k}_{\sigma_{\ell, 1}}$ and some discrete subset $D$. We can assume that $\zeta^{\prime}$ is a $\varrho$-semi-canonical element. The corresponding $S^{1}$-invariant solution $u_{\zeta} \circ \Phi$ takes values in $\Omega_{\xi}\left(S U(2 n)^{\sigma_{\ell, 1}}\right)$, with $\xi \in \mathfrak{I}^{\prime}\left(S U(2 n)^{\sigma_{\varrho, 1}}\right)$; and both $\Phi_{-1}$ and $\left(u_{\zeta} \circ \Phi\right)_{-1}$ take values in $P_{\xi}^{\sigma_{e}, 1}$. A priori, $\xi$ can be different from $\zeta$ since $\sigma_{\varrho, 1}$ is not a fundamental outer involution. However, by Lemma 25 we have $P_{\xi}^{\sigma_{Q}, 1}=P_{e}^{\sigma_{Q, 1}}=P_{\zeta^{\prime}, 1}^{\sigma^{\prime}}$.

If $\zeta$ is a $\varrho$-canonical element such that $\zeta^{\prime} \preceq \zeta$ and $\mathcal{U}_{\zeta^{\prime}, \zeta^{\prime}-\zeta}(\Phi)$ is constant, then, taking into account Proposition 15. there exists a $T_{\tau}$-invariant extended solution $\tilde{\Phi}: S^{2} \backslash D \rightarrow U_{\zeta}^{\tau}(S U(2 n))$, where

$$
\tau=\operatorname{Ad}\left(\exp \pi\left(\zeta^{\prime}-\zeta\right)\right) \circ \sigma_{\varrho, 1} .
$$

such that $\tilde{\Phi}_{-1}$ take values in $P_{\zeta}^{\tau}$ and $\varphi$ is given up to isometry by

$$
\varphi=\exp \left(\zeta^{\prime}-\zeta\right) \tilde{\Phi}_{-1} \tau_{0} .
$$

We conclude that, given a pair $(\zeta, \tau)$, where $\zeta \in \mathfrak{I}\left(S U(2 n)^{\sigma_{\varrho}}\right)$ is a $\varrho$-canonical element and $\tau$ is an outer involution of the form (42), any extended solution $\left.\tilde{\Phi}: S^{2} \backslash D \rightarrow U_{\zeta}^{\tau}(S U(2 n))\right)$ gives rise via (43) to an harmonic map $\varphi$ from the two-sphere into $\mathcal{L}_{2 n}^{s}$ and, conversely, all harmonic two-spheres into $\mathcal{L}_{2 n}^{s}$ arise in this way.

For $\mathcal{L}_{4}^{s}$, since $\exp \pi 2 \zeta_{1}$ belongs to the centre of $S U(4)$, we have five classes of harmonic maps into $\mathcal{L}_{4}^{s}$ :

$$
\left(2 \zeta_{1}, \sigma_{\varrho, 1}\right),\left(\zeta_{2}, \sigma_{\varrho, 1}\right),\left(2 \zeta_{1}+\zeta_{2}, \sigma_{\varrho, 1}\right)\left(\zeta_{2}, \operatorname{Ad} \exp \pi \zeta_{2} \circ \sigma_{\varrho, 1}\right),\left(2 \zeta_{1}+\zeta_{2}, \operatorname{Ad} \exp \pi \zeta_{2} \circ \sigma_{\varrho, 1}\right) .
$$

Let us consider in detail the class $\left(\zeta_{2}, \sigma_{\varrho, 1}\right)$. Clearly $r\left(\zeta_{2}\right)=2$. Let $W_{1}, W_{2}, W_{3}$ and $W_{4}$ be the complex onedimensional images of $E_{1}, E_{2}, E_{3}$ and $E_{4}$, respectively. That is, $W_{i}=\operatorname{Span}\left\{v_{i}\right\}$, where $v_{i}$ are defined by (41). Any extended solution $\Phi: S^{2} \backslash D \rightarrow U_{\zeta_{2}}^{\sigma_{Q}, 1}$ is given by $\Phi=\exp C \cdot \gamma_{\zeta_{2}}$, with $\gamma_{\zeta_{2}}(\lambda)=\lambda^{-1} \pi_{W_{4}}+\pi_{W_{3} \oplus W_{2}}+\lambda \pi_{W_{1}}$, for some holomorphic vector-valued function $C: S^{2} \backslash D \rightarrow\left(\mathfrak{u}_{\zeta_{2}}^{0}\right)_{\sigma_{\ell, 1}}$, where

$$
\left(\mathfrak{u}_{\zeta_{2}}^{0}\right)_{\sigma_{Q, 1}}=\left(\mathfrak{p}_{0}^{\zeta_{2}}\right)^{\perp} \cap \mathfrak{k}_{\sigma_{\varrho, 1}}^{\mathbb{C}}+\lambda\left(\mathfrak{p}_{1}^{\zeta_{2}}\right)^{\perp} \cap \mathfrak{m}_{\sigma_{\varrho, 1}}^{\mathbb{C}}
$$


and

$$
\begin{aligned}
\left(\mathfrak{p}_{0}^{\zeta_{2}}\right)^{\perp} \cap \mathfrak{k}_{\sigma_{Q, 1}}^{\mathbb{C}} & =\left(\mathfrak{g}_{L_{1}-L_{2}} \oplus \mathfrak{g}_{L_{3}-L_{4}} \oplus \mathfrak{g}_{L_{1}-L_{3}} \oplus \mathfrak{g}_{L_{2}-L_{4}}\right) \cap \mathfrak{k}_{\sigma_{\ell, 1}}^{\mathbb{C}}, \\
\left(\mathfrak{p}_{1}^{\zeta_{2}}\right)^{\perp} \cap \mathfrak{m}_{\sigma_{Q, 1}}^{\mathbb{C}} & =\mathfrak{g}_{L_{1}-L_{4}} \cap \mathfrak{m}_{\sigma_{\ell, 1}}^{\mathbb{C}}=\mathfrak{g}_{L_{1}-L_{4}} .
\end{aligned}
$$

We have $\sigma_{\varrho, 1}\left(X_{1,2}\right)=-X_{3,4}$ and $\sigma_{\varrho, 1}\left(X_{1,3}\right)=X_{2,4}$. Hence we can write $C=C_{0}+C_{1} \lambda$, with

$$
C_{0}=a\left(X_{1,2}-X_{3,4}\right)+b\left(X_{1,3}+X_{2,4}\right), \quad C_{1}=c X_{1,4}
$$

for some meromorphic functions $a, b, c$ on $S^{2}$. The harmonicity equations impose that $a b^{\prime}-b a^{\prime}=0$, which means that $b=\alpha a$ for some constant $\alpha \in \mathbb{C}$. Hence given arbitrary meromorphic functions $a, c$ on $S^{2}$ and a complex constant $\alpha$,

$$
\Phi=\left(\begin{array}{cccc}
1 & a & \alpha a & c \lambda \\
0 & 1 & 0 & -\alpha a \\
0 & 0 & 1 & a \\
0 & 0 & 0 & 1
\end{array}\right) \cdot \gamma_{\zeta_{1}}
$$

is an extended solution associated to some harmonic map in the class $\left(\zeta_{2}, \sigma_{\varrho, 1}\right)$. Reciprocally, any harmonic map in such class arises in this way.

\section{REFERENCES}

[1] F. E. Burstall and M. A. Guest, Harmonic two-spheres in compact symmetric spaces, revisited, Math. Ann. 309 (1997), no. 4, $541-572$.

[2] F. E. Burstall, J. H. Rawnsley, Twistor Theory for Riemannian Symmetric Spaces, Lectures Notes in Math. 1424 Berlin, Heidelberg: 1990.

[3] E. Calabi, Minimal immersions of surfaces in Euclidean spheres, J. Diff. Geom., 1 (1967), 111-125.

[4] N. Correia and R. Pacheco, Harmonic maps of finite uniton number into $G_{2}$, Math. Z. 271 (2012), no. 1-2, 13-32.

[5] N. Correia and R. Pacheco, Extended Solutions of the Harmonic Map Equation in the Special Unitary Group, Q. J. Math. 65 (2014), no. 2, 637-654.

[6] N. Correia and R. Pacheco, Harmonic maps of finite uniton number and their canonical elements, Ann. Global Anal. Geom. 47 (2015), no. 4, 335-358.

[7] J. Dorfmeister, F. Pedit and H. Wu, Weiestrass type representation of harmonic maps into symmetric spaces, Comm. Anal. Geom. 6 (1998), 633-668.

[8] J.-H. Eschenburg, A.-L. Mare, and P. Quast, Pluriharmonic maps into outer symmetric spaces and a subdivision of Weyl chambers, Bull. London Math. Soc. 42 (2010), no. 6, 1121-1133.

[9] J. Eells and J. C. Wood, Harmonic maps from surfaces to complex projective spaces, Adv. in Math. 49 (1983), no. 3, $217-263$.

[10] W. Fulton and J. Harris, Representation theory (a first course), Graduate Texts in Mathematics, vol. 129, Springer-Verlag, New York, 1991.

[11] M. A. Guest and Y. Ohnita, Loop group actions on harmonic maps and their applications, Harmonic maps and integrable systems, 273-292, Aspects Math., E23, Vieweg, Braunschweig, 1994.

[12] S. Helgason, Differential Geometry, Lie Groups, and Symmetric Spaces, Academic Press, New York 1978.

[13] H. Ma, Explicit construction of harmonic two-spheres in $S U(3) / S O(3)$, Kyushu J. Math. 55 (2001), $237-247$.

[14] S. Murakami, Sur la classification des algèbres de Lie réelles et simples, Osaka J. Math. 2 (1965), $291-307$.

[15] A.N. Pressley and G.B. Segal, Loop Groups, Oxford University Press, 1986.

[16] A. Bahy-El-Dien and J.C. Wood, The explicit construction of all harmonic two-spheres in $G_{2}\left(\mathbb{R}^{n}\right)$, J. Reine Angew. Math. 398 (1989), 36-66.

[17] K. Uhlenbeck, Harmonic maps into Lie groups (classical solutions of the chiral model), J. Diff. Geom. 30 (1989), 1-50.

[18] W. Ziller, W. Lie groups, representation theory and symmetric spaces. Notes for a course given in the fall of 2010 at the University of Pennsylvania and 2012 at IMPA.

Universidade da Beira Interior, Rua Marquês d’Ávila e Bolama, 6200-001 Covilhã, Portugal

E-mail address: ncorreia@ubi.pt, rpacheco@ubi.pt 